\title{
CHAINS, ANTICHAINS, AND COMPLEMENTS IN INFINITE PARTITION LATTICES
}

\author{
JAMES EMIL AVERY, JEAN-YVES MOYEN, PAVEL RUZICKA, \\ AND JAKOB GRUE SIMONSEN
}

\begin{abstract}
We consider the partition lattice $\Pi_{\kappa}$ on any set of transfinite cardinality $\kappa$ and properties of $\Pi_{\kappa}$ whose analogues do not hold for finite cardinalities. Assuming the Axiom of Choice we prove: (I) the cardinality of any maximal well-ordered chain is always exactly $\kappa$; (II) there are maximal chains in $\Pi_{\kappa}$ of cardinality $>\kappa$; (III) if, for every cardinal $\lambda<\kappa$, we have $2^{\lambda}<2^{\kappa}$, there exists a maximal chain of cardinality $<2^{\kappa}$ (but $\geq \kappa$ ) in $\Pi_{2^{\kappa}}$; (IV) every non-trivial maximal antichain in $\Pi_{\kappa}$ has cardinality between $\kappa$ and $2^{\kappa}$, and these bounds are realized. Moreover we can construct maximal antichains of cardinality $\max \left(\kappa, 2^{\lambda}\right)$ for any $\lambda \leq \kappa ;(\mathrm{V})$ all cardinals of the form $\kappa^{\lambda}$ with $0 \leq \lambda \leq \kappa$ occur as the number of complements to some partition $\mathcal{P} \in \Pi_{\kappa}$, and only these cardinalities appear. Moreover, we give a direct formula for the number of complements to a given partition; (VI) Under the Generalized Continuum Hypothesis, the cardinalities of maximal chains, maximal antichains, and numbers of complements are fully determined, and we provide a complete characterization.
\end{abstract}

Let $\kappa$ be a cardinal and let $S$ be a set of cardinality $\kappa$. The set of partitions of $S$ forms a lattice when endowed with the binary relation $\leq$, called refinement, defined by $\mathcal{P} \leq \mathcal{Q}$ if and only if each block of $\mathcal{P}$ is a subset of a block of $\mathcal{Q}$. This lattice is called the partition lattice on $S$, and is denoted $\Pi(S)$. By the standard correspondence between partitions and equivalence relations, it follows that $\Pi(S)$ is isomorphic to the lattice $\operatorname{Equ}(S)$ of equivalence relations on $S$ ordered by set inclusion on $S \times S$.

As the particulars of $S$ do not affect the order-theoretic properties of $\Pi(S)$ we shall without loss of generality restrict our attention to the lattice $\Pi_{\kappa}=\Pi(\kappa)$. Initiated by a seminal paper by Ore Ore42, many of the properties of $\Pi_{\kappa}$ that hold for arbitrary cardinals $\kappa$ are well-known. Indeed, it is known that $\Pi_{\kappa}$ is complete, matroid (hence atomistic and semimodular), non-modular (hence non-distributive) for $\kappa \geq 4$, relatively complemented (hence complemented), and simple [Bir40, §8-9], RS92, Grä03, Sec. IV.4].

For properties depending on $\kappa$, only a few results exist in the literature for infinite $\kappa$. Czédli has proved that if there is no inaccessible cardinal $\leq \kappa$ then the following holds: If $\kappa \geq 4, \Pi_{\kappa}$ is generated by four elements Czé96a, and if $\kappa \geq 7, \Pi_{\kappa}$ is $(1+1+2)$-generated [Czé99] (for $\kappa=\aleph_{0}$, slightly stronger results hold

James Avery: Niels Bohr Institute, University of Copenhagen, Blegdamsvej 17, 2100 Copenhagen $\varnothing$, Denmark, E-Mail: avery@diku.dK

Jean-Yves Moyen: Laboratoire d'Informatique de Paris Nord, Université Paris XiII, 99, avenue J.-B. Clément, 93430 Villetaneuse, France, E-Mail: JEAN-YVES . MOYEN@LIPN . UN IV-PARIS13. FR

Pavel Ruzicka: Department of Algebra , Room 307, Sokolovska 83, 186 75, Prague, Czech Republic, E-Mail: Ruzicka@karlin.mfF.cuni.cz

Jakob Grue Simonsen: Department of Computer Science, University of Copenhagen (DIKU), Nualsgade 128-132, 2300 Copenhagen S, Denmark, E-Mail: simonsen@diku.dK

1991 Mathematics Subject Classification. Primary 06B05; 06C15.

Key words and phrases. Partition lattice; order theory; antichain; chain; complement; orthocomplemented lattice; cardinal; ordinal. 
Czé96b]). It appears that no further results are known, beyond those holding for all cardinalities, finite or infinite. The aim of the present work is to prove a number of results concerning $\Pi_{\kappa}$ that depend on $\kappa$ being an infinite cardinal.

\section{PREliminaries And NOTAtion}

We work in ZF with the Axiom of Choice (AC). As usual, a set $S$ is well-ordered if and only if it is totally ordered and every non-empty subset of $S$ has a least element. Throughout the paper, we use von Neumann's characterization of ordinals: a set $S$ is an ordinal if and only if it is strictly well-ordered by $\subsetneq$ and every element of $S$ is a subset of $S$. The order type of a well-ordered set $S$ is the (necessarily unique) ordinal $\alpha$ that is order-isomorphic to $S$. Cardinals and ordinals are denoted by Greek letters $\alpha, \beta, \gamma, \delta, \ldots$ for ordinals and $\kappa, \lambda, \ldots$ for cardinals. We denote by $\omega_{\kappa}$ the initial ordinal of $\kappa$, and by $|\alpha|$ the cardinality of $\alpha$. The cardinality of a set is denoted $|S|$ and its powerset is denoted $\mathscr{P}(S)$. For a cardinal $\kappa$, we denote by $\kappa^{+}$ its successor and by $\kappa^{-}$its predecessor cardinal. Note that $\kappa^{-}$is defined only if $\kappa$ is a successor cardinal.

Many standard results on cardinal arithmetic can be found in [HSW99, among other places, and are used frequently throughout the proofs.

Recall that a chain in a poset $(\mathbf{P}, \leq)$ is a subset of $\mathbf{P}$ that is totally ordered by $\leq$. Similarly, an antichain in $(\mathbf{P}, \leq)$ is a subset of $\mathbf{P}$ such that any two distinct elements of the subset are $\leq$-incomparable. A chain (respectively, antichain) in $(\mathbf{P}, \leq)$ is maximal if no element of $\mathbf{P}$ can be added to the chain without losing the property of being a chain (respectively, antichain). Observe that if $\mathbf{P}$ contains a bottom element, $\perp$, or a top element, $T$, it belongs to any maximal chain. A chain $\mathbf{C}$ in $(\mathbf{P}, \leq)$ is saturated if, for any two elements $\mathcal{Q}<\mathcal{S}$ of the chain, there is no element $\mathcal{R} \in \mathbf{P} \backslash \mathbf{C}$ such that $\mathcal{Q}<\mathcal{R}<\mathcal{S}$ and $\mathbf{C} \cup\{\mathcal{R}\}$ is a chain; notably, a chain containing $\perp$ and $T$ is maximal if and only if it is saturated. We say that a chain is endpoint-including if it contains a least and a greatest element, not necessarily equal to $\perp$ and $T$, respectively. By the Maximal Chain Theorem [Hau14, every chain in a poset is contained in a maximal chain.

We denote partitions (and equivalences) of $\kappa$ by capital italic Roman letters $\mathcal{P}, \mathcal{Q}, \ldots$, and denote subsets of $\Pi_{\kappa}$ such as chains and antichains by capital boldface letters $\mathbf{C}, \mathbf{D}, \ldots$; If $\mathcal{P}=\left\{B_{\delta}\right\}$ is a partition, we call its elements, $B_{\delta}$, blocks. It is easily seen that $\perp=\{\{\gamma\} \mid \gamma \in \kappa\}$ and $T=\{\kappa\}$; that is, the set of all singleton subsets of $\kappa$, respectively the singleton set containing all elements of $\kappa$.

As is usual, if $\mathcal{P}, \mathcal{Q} \in \Pi_{\kappa}$, we write $\mathcal{P} \prec \mathcal{Q}$ if $\mathcal{P}<\mathcal{Q}$ and no $\mathcal{R} \in \Pi_{\kappa}$ exists such that $\mathcal{P}<\mathcal{R}<\mathcal{Q}$. Furthermore, $\mathcal{P} \preceq \mathcal{Q}$ denotes that either $\mathcal{P} \prec \mathcal{Q}$ or $\mathcal{P}=\mathcal{Q}$. It follows that $\mathcal{P} \prec \mathcal{Q}$ if and only if $\mathcal{Q}$ can be obtained by merging exactly two distinct blocks of $\mathcal{P}$. If $\mathbf{X}$ is a subset of $\Pi_{\kappa}$, we write $\mathcal{P} \prec_{\mathbf{X}} \mathcal{Q}$ if $\mathcal{P}, \mathcal{Q} \in \mathbf{X}$ with $\mathcal{P}<\mathcal{Q}$, and there exists no $\mathcal{R} \in \mathbf{X}$ such that $\mathcal{P}<\mathcal{R}<\mathcal{Q}$. A subset $\mathbf{X} \subseteq \Pi_{\kappa}$ is called covering if $\mathcal{P} \prec \mathbf{x} \mathcal{Q}$ implies $\mathcal{P} \prec \mathcal{Q}$.

A block $B$ induces an equivalence relation on $\kappa$, defined by $\delta \equiv_{B} \gamma$ if and only if both $\delta, \gamma \in B$, and a partition $\mathcal{P}$ naturally induces an equivalence relation defined by $\delta \equiv_{\mathcal{P}} \gamma$ if and only if there is a block $B \in \mathcal{P}$ with $\delta \equiv_{B} \gamma$. Conversely, any equivalence relation corresponds to the partition whose blocks are the maximal sets of equivalent elements. This one-to-one correspondence allows us to consider a partition as its corresponding equivalence relation when convenient, and vice versa.

If $\mathcal{P} \in \Pi_{\kappa}$ contains exactly one block $B$ with $|B| \geq 2$ and the remaining blocks are all singletons, we call $\mathcal{P}$ a singular partition, following Ore Ore42.

If $\mathbf{C} \subseteq \Pi_{\kappa}$, then its greatest lower bound $\wedge \mathbf{C}$ is the partition that satisfies $x \equiv_{\wedge} \mathbf{C} y$ if and only if $x \equiv_{\mathcal{P}} y$ for all $\mathcal{P} \in \mathbf{C}$. That is, the blocks of $\wedge \mathbf{C}$ are all the nonempty intersections whose terms are exactly one block from every partition 
$\mathcal{P} \in \mathbf{C}$. Conversely, its least upper bound $\vee \mathbf{C}$ is the partition such that $\gamma \equiv{ }_{{ }_{\mathbf{C}}} \delta$ if and only if there exists a finite sequence of partitions $\mathcal{P}^{1}, \ldots, \mathcal{P}^{k} \in \mathbf{C}$ and elements $\beta^{0}, \ldots, \beta^{k}, \in \kappa$ such that $\gamma=\beta^{0} \equiv_{\mathcal{P}^{1}} \beta^{1} \equiv_{\mathcal{P}^{2}} \beta^{2} \equiv_{\mathcal{P}^{3}} \cdots \equiv_{\mathcal{P}^{k}} \beta^{k}=\delta$. A set of partitions is called complete if it contains both the least upper bound and greatest lower bound of all its subset, and closed if this is true for every nonempty subset, i.e., a closed set need not include $\perp$ and $T$.

Finally, the cofinality $\mathrm{cf}(\kappa)$ of an infinite cardinal $\kappa$ is the least cardinal $\lambda$ such that a set of cardinality $\kappa$ can be written as a union of $\lambda$ sets of cardinality strictly smaller than $\kappa: \operatorname{cf}(\kappa)=\min \left\{|I||\kappa=| \bigcup_{i \in I} A_{i}|\wedge \forall i \in I,| A_{i} \mid<\kappa\right\}$. Since $\kappa=$ $\bigcup_{i \in \kappa}\{i\}$, we always have $\operatorname{cf}(\kappa) \leq \kappa$.

If $\operatorname{cf}(\kappa)=\kappa$, then the cardinal $\kappa$ is called regular, otherwise it is called singular. Under AC, which is assumed throughout this paper, every infinite successor cardinal is regular. König's Theorem Kön05 implies $\mathrm{cf}\left(2^{\kappa}\right)>\kappa$, and we additionally have $2^{\kappa} \leq 2^{\lambda}$ whenever $\kappa<\lambda$. By Easton's theorem [Eas70], these are the only two constraints on permissible values for $2^{\kappa}$ when $\kappa$ is regular and when only ZFC is assumed. In contrast, when the Generalized Continuum Hypothesis (GCH) is assumed, cardinal exponentiation is completely determined.

For infinite $\kappa, \Pi_{\kappa}$ has cardinality $2^{\kappa}$ which provides a weak upper bound on the cardinality of its subsets, in particular maximal chains, maximal antichains, and sets of complements.

\section{Results}

We summarize here the main contributions of the paper.

Theorem (Well-ordered chains: Theorem 4.4). Let $\kappa$ be any cardinal. The cardinality of a maximal well-ordered chain in $\Pi_{\kappa}$ is always exactly $\kappa$.

Theorem (Long chains: Theorem 5.2). Let $\kappa$ be an infinite cardinal. There exist chains of cardinality $>\kappa$ in $\Pi_{\kappa}$.

Theorem (Short chains: Theorem 6.14). Let $\kappa$ be an infinite cardinal such that for every cardinal $\lambda<\kappa$ we have $2^{\lambda}<2^{\kappa}$. Then there exists a maximal chain of cardinality $<2^{\kappa}($ but $\geq \kappa)$ in $\Pi_{2^{\kappa}}$.

Theorem (Antichains: Theorems 7.1 and 7.3). Let $\kappa$ be an infinite cardinal. Each non-trivial maximal antichain in $\Pi_{\kappa}$ has cardinality between $\kappa$ and $2^{\kappa}$, and these bounds are tight (there exists maximal antichains with each of these two cardinalities).

Theorem (Complements: Theorems 8.7, 8.9, and 9.7). Let $\kappa$ be an infinite cardinal. All cardinals of the form $\kappa^{\lambda}$ with $0 \leq \lambda \leq \kappa$ occur as the number of complements to some partition $\mathcal{P} \in \Pi_{\kappa}$, and these are the only cardinalities the set of complements can have.

For non-trivial partitions $\mathcal{P} \notin\{\perp, \top\}$, the number of complements is between $\kappa$ and $2^{\kappa}$, i.e. $\kappa^{\lambda}$ with $1 \leq \lambda \leq \kappa$. The number of complements to $\mathcal{P}$ is $2^{\kappa}$ unless (i) $\mathcal{P}$ contains exactly one block $B$ of cardinality $\kappa$, and (ii) $|\kappa \backslash B|<\kappa$. If $\mathcal{P}$ contains one block $B$ of size $\kappa$, then $\mathcal{P}$ has $\kappa^{|\kappa \backslash B|}$ complements.

Theorem (Full characterizations under GCH, Theorem 9.8). Under the Generalized Continuum Hypothesis, when $\kappa$ is an infinite cardinal:

(1) Any maximal well-ordered chain in $\Pi_{\kappa}$ always has cardinality $\kappa$.

(2) Any general maximal chain in $\Pi_{\kappa}$ has cardinality

(a) $\kappa^{-}, \kappa$, or $\kappa^{+}$(and all three are always achieved) if $\kappa$ is a successor cardinal; and

(b) either $\kappa$ or $\kappa^{+}$(and both are achieved) if $\kappa$ is a limit cardinal. 
(3) Any non-trivial maximal antichain in $\Pi_{\kappa}$ has cardinality either $\kappa$ or $\kappa^{+}$, and both are achieved.

(4) Any non-trivial partition has either $\kappa$ or $\kappa^{+}$complements. $\mathcal{P} \notin\{\perp, \top\}$ has $\kappa$ complements if and only if (i) $\mathcal{P}$ contains exactly one block, $B$, of cardinality $\kappa$, and (ii) $|\kappa \backslash B|<\operatorname{cf}(\kappa)$; otherwise, $\mathcal{P}$ has $\kappa^{+}$complements.

\section{SOME BASIC PROPERTIES}

3.1. Saturated chains in complete lattices. In this section, we prove a few properties of chains on complete lattices (not necessarily the partition lattice) that will be used in the later sections.

Definition 3.1. Let $\mathbf{C}$ be a chain in a complete lattice $\mathbf{L}$. Define the lower, respectively upper, subchain relative to $x \in \mathbf{L}$ as $\mathbf{C}_{x}^{-}=\{y \in \mathbf{C} \mid y<x\}$ and $\mathbf{C}_{x}^{+}=\{y \in \mathbf{C} \mid x<y\}$.

Lemma 3.2. Let $\mathbf{C}$ be a closed chain in a complete lattice $\mathbf{L}$, and $x \in \mathbf{L}$ such that $\mathbf{C} \cup\{x\}$ is a chain, and the sets $\mathbf{C}_{x}^{-}$and $\mathbf{C}_{x}^{+}$are nonempty.

- If $x \notin \mathbf{C}$, then $\vee \mathbf{C}_{x}^{-} \prec \mathbf{C} \wedge \mathbf{C}_{x}^{+}$.

- If $x \in \mathbf{C}$, then either (i) $\vee \mathbf{C}_{x}^{-}=x=\wedge \mathbf{C}_{x}^{+}$, (ii) $\vee \mathbf{C}_{x}^{-} \prec \mathbf{C} \wedge \mathbf{C}_{x}^{+}$, or (iii) $\vee \mathbf{C}_{x}^{-} \prec_{\mathbf{C}} x \prec_{\mathbf{C}} \wedge \mathbf{C}_{x}^{+}$.

This also implies that when $x \in \mathbf{C}, \vee \mathbf{C}_{x}^{-} \preceq_{\mathbf{C}} x \preceq_{\mathbf{C}} \wedge \mathbf{C}_{x}^{+}$.

This is easily proved by checking each case. The key points are: i) Because the chain $\mathbf{C}$ is closed, there exists no $y \in \mathbf{C}$ with $\vee \mathbf{C}_{x}^{-}<y<\wedge \mathbf{C}_{x}^{+}$; and ii) $x \in \mathbf{C} \Rightarrow \vee \mathbf{C}_{x}^{-} \leq x \leq \wedge \mathbf{C}_{x}^{+}$.

Lemma 3.3. An endpoint-including chain $\mathbf{C}$ in a complete lattice $\mathbf{L}$ is saturated if and only if it is closed and covering; and it is maximal if and only if it is complete and covering.

Proof. Let in the following $\mathbf{C}$ be a chain in a complete lattice $\mathbf{L}$, such that $\mathbf{C}$ has minimal element $c_{\min }$ and maximal element $c_{\max }$. Since $\mathbf{C}$ is totally ordered, $c_{\text {min }}=\wedge \mathbf{C}$ is its unique least element, and $c_{\max }=\vee \mathbf{C}$ is its unique greatest element.

Saturated implies closed. Assume that there exists a nonempty subset $\mathbf{D} \subseteq \mathbf{C}$ with greatest lower bound $\wedge \mathbf{D} \notin \mathbf{C}$. By construction,

$$
c_{\min }=\wedge \mathbf{C}<\wedge \mathbf{D}<\vee \mathbf{C}=c_{\max }
$$

Let $x$ be an arbitrary element of $\mathbf{C}$. Since $\mathbf{C}$ is a chain, either $d \leq x$ for some $d \in \mathbf{D}$, which implies $\wedge \mathbf{D} \leq x$; or $x<d$ for all $d \in \mathbf{D}$ which implies $x \leq \wedge \mathbf{D}$. It follows that $\mathbf{C} \cup \wedge \mathbf{D}$ is a chain. This together with Equation (3.1) contradicts that $\mathbf{C}$ is saturated. Dually we prove that $\vee \mathbf{D} \in \mathbf{C}$ for every nonempty $\mathbf{D} \subseteq \mathbf{C}$. Hence C is saturated only if it is closed.

Saturated implies covering. Assume $\mathbf{C}$ is not covering, i.e. there exists $x, z \in$ $\mathbf{C}$ with $x \prec_{\mathbf{C}} z$ but $x \nprec_{\mathbf{L}} z$. The second relation implies that there exists $y \in \mathbf{L} \backslash \mathbf{C}$ with $x<y<z$. Because $x \prec_{\mathbf{C}} z$, every other element of $\mathbf{C}$ is either smaller than $x$ or larger than $z$, hence comparable with $y$, whereby $\mathbf{C} \cup\{y\}$ is a chain. Hence $\mathbf{C}$ is saturated only if it is covering.

Closed and covering implies saturated. Assume that $\mathbf{C}$ is closed and covering, and choose any $x \in \mathbf{L}$ with $c_{\min }<x<c_{\max }$ for which $\mathbf{C} \cup\{x\}$ is still a chain. Then $c_{\min } \in \mathbf{C}_{x}^{-}$and $c_{\max } \in \mathbf{C}_{x}^{+}$, so both sets are nonempty. If $x \notin \mathbf{C}$, Lemma 3.2 implies $\vee \mathbf{C}_{x}^{-} \prec_{\mathbf{C}} \wedge \mathbf{C}_{x}^{+}$, and, because $\mathbf{C}$ is covering, $\vee \mathbf{C}_{x}^{-} \prec \wedge \mathbf{C}_{x}^{+}$. But $\vee \mathbf{C}_{x}^{-} \leq x \leq \wedge \mathbf{C}_{x}^{+}$, so this implies $x=\vee \mathbf{C}_{x}^{-}$or $x=\wedge \mathbf{C}_{x}^{+}$, contradicting $x \notin \mathbf{C}$, as both are in $\mathbf{C}$. Hence, $\mathbf{C}$ is saturated.

Maximal is equivalent to complete and covering. A maximal chain is a saturated chain that contains $T$ and $\perp$; and a complete sublattice is a closed sublattice that contains $T$ and $\perp$, yielding the lemma's second statement. 


\subsection{Meets and joins of chains in $\Pi_{\kappa}$.}

Definition 3.4. Let $\mathbf{C}$ be a chain in $\Pi_{\kappa}$. We define the lower and upper subchains relative to $x, y \in \kappa$ as $\mathbf{C}_{x, y}^{-}=\left\{\mathcal{P} \in \mathbf{C} \mid x \equiv_{\mathcal{P}} y\right\}$ and $\mathbf{C}_{x, y}^{+}=\left\{\mathcal{P} \in \mathbf{C} \mid x \equiv_{\mathcal{P}} y\right\}$

Lemma 3.5. Let $\mathbf{C}$ be a chain in $\Pi_{\kappa}$ and $\mathbf{D}$ be any set of $\kappa$-partitions.

(1) $x \equiv \wedge \mathbf{D} y$ if and only if $x \equiv \mathcal{P} y$ for all $\mathcal{P} \in \mathbf{D}$;

(2) $x \equiv_{\vee_{\mathbf{C}}} y$ if and only if $x \equiv_{\mathcal{P}} y$ for some $\mathcal{P} \in \mathbf{C}$;

(3) If $\mathbf{C}$ is closed, given elements $x, y \in \kappa$ for which $\mathbf{C}_{x, y}^{-}$and $\mathbf{C}_{x, y}^{+}$are both nonempty, we have
(a) $\wedge \mathbf{C}_{x, y}^{+} \in \mathbf{C}_{x, y}^{+}$,
(b) $\vee \mathbf{C}_{x, y}^{-} \in \mathbf{C}_{x, y}^{-}$,
(c) $\vee \mathbf{C}_{x, y}^{-} \prec \mathbf{C} \wedge \mathbf{C}_{x, y}^{+}$(and, if $\mathbf{C}$ is covering, $\vee \mathbf{C}_{x, y}^{-} \prec \wedge \mathbf{C}_{x, y}^{+}$).

Proof.

(1) This is the definition of $\wedge \mathbf{D}$.

(2) By definition of $x \equiv_{\vee \mathbf{C}} y$, there exist finite sequences $\left\{x^{i}\right\}$ and $\left\{\mathcal{P}^{i}\right\}$ such that $x=x^{0} \equiv_{\mathcal{P}^{1}} x^{1} \equiv_{\mathcal{P}^{2}} x^{2} \equiv_{\mathcal{P}^{3}} \cdots \equiv_{\mathcal{P}^{n}} x^{n}=y$. But because $\mathbf{C}$ is totally ordered, the finite set $\left\{\mathcal{P}^{i}\right\} \subset \mathbf{C}$ has a greatest element $\mathcal{P}^{k}$, and thus $x_{0} \equiv_{\mathcal{P}^{k}} x_{1} \equiv_{\mathcal{P}^{k}} \cdots \equiv_{\mathcal{P}^{k}} x_{n}$, whereby $x \equiv_{\mathcal{P}^{k}} y$.

(3) (a) follows immediately from (1), and (b) from the negation of (2). For (c), notice that (a) and (b) imply $\vee \mathbf{C}_{x, y}^{-} \neq \wedge \mathbf{C}_{x, y}^{+}$, whereby $\vee \mathbf{C}_{x, y}^{-}<\wedge \mathbf{C}_{x, y}^{+}$. Because every $\mathcal{P} \in \mathbf{C}$ lies either in $\mathbf{C}_{x, y}^{-}$or $\mathbf{C}_{x, y}^{+}$, no $\mathcal{P} \in \mathbf{C}$ can have the property $\vee \mathbf{C}_{x, y}^{-}<\mathcal{P}<\wedge \mathbf{C}_{x, y}^{+}$, whereby $\vee \mathbf{C}_{x, y}^{-} \prec_{\mathbf{C}} \wedge \mathbf{C}_{x, y}^{+}$. The final statement is simply the definition of covering.

Corollary 3.6. Given a non-empty chain $\mathbf{C}$, each block of $\vee \mathbf{C}$ is the union, and each block of $\wedge \mathbf{C}$ is the intersection, of an increasing sequence of blocks, one from each $\mathcal{P} \in \mathbf{C}$.

Proof. Fix $x \in \kappa$, and for each $\mathcal{P} \in \mathbf{C}$ let $B_{\mathcal{P}}$ be the block of $\mathcal{P}$ containing $x$. Let $B$ (resp. $B^{\prime}$ ) be the block of $\wedge \mathbf{C}$ (resp. $\vee \mathbf{C}$ ) that contain $x$. The second equivalence in each case are Lemma $3.5(1-2)$. The statement for the greatest lower bound is derived as

$$
y \in B \Leftrightarrow x \equiv \wedge \mathbf{C} y \Leftrightarrow \forall \mathcal{P} \in \mathbf{C}, x \equiv_{\mathcal{P}} y \Leftrightarrow \forall \mathcal{P} \in \mathbf{C}, y \in B_{\mathcal{P}} \Leftrightarrow y \in \bigcap_{\mathcal{P} \in \mathbf{C}} B_{\mathcal{P}}
$$

and for the least upper bound as

$$
y \in B^{\prime} \Leftrightarrow x \equiv_{\vee_{\mathbf{C}}} y \Leftrightarrow \exists \mathcal{P} \in \mathbf{C}, x \equiv_{\mathcal{P}} y \Leftrightarrow \exists \mathcal{P} \in \mathbf{C}, y \in B_{\mathcal{P}} \Leftrightarrow y \in \bigcup_{\mathcal{P} \in \mathbf{C}} B_{\mathcal{P}}
$$

Notice that the choice of $x$, hence also of the $B_{\mathcal{P}}$ in the union, is far from unique. These lemmas essentially state that "nothing happens when going to the limit". All the equivalences between elements that are present in the limit (e.g., the join) were actually already here in some partition of the chain. Notably, if the chain is well-ordered, the "merge" between two elements must happen between a (partition indexed by an) ordinal and its successor, and does not suddenly appear at a (partition indexed by a) limit ordinal.

\subsection{Restriction.}

Definition 3.7 (Restriction). Given a partition $\mathcal{P}$ of $\kappa$, we define its restriction to $\lambda \leq \kappa$ as $\mathcal{P} \sqcap \lambda=\{B \cap \lambda \mid B \in \mathcal{P}\} \backslash\{\emptyset\}$, which is a partition of $\lambda$. Similarly, a set $\mathbf{D}$ of partitions restricts to a subset of $\Pi_{\lambda}$ as $\mathbf{D} \sqcap \lambda=\{\mathcal{P} \sqcap \lambda \mid \mathcal{P} \in \mathbf{D}\}$. 
Lemma 3.8. (i) $\top_{\kappa} \sqcap \lambda=\top_{\lambda}$; (ii) $\perp_{\kappa} \sqcap \lambda=\perp_{\lambda}$; (iii) $\mathcal{P}<\mathcal{Q}$ implies $\mathcal{P} \sqcap \lambda \leq \mathcal{Q} \sqcap \lambda$; (iv) if $\mathbf{C}$ is a chain, so is $\mathbf{C} \sqcap \lambda$; (v) if $\mathcal{P}, \mathcal{Q}$ are comparable, then $\mathcal{P} \sqcap \lambda<\mathcal{Q} \sqcap \lambda$ implies $\mathcal{P}<\mathcal{Q}$; and (vi) $\mathcal{P} \prec \mathcal{Q}$ implies $\mathcal{P} \sqcap \lambda \preceq \mathcal{Q} \sqcap \lambda$.

All these Facts can be checked easily by direct applications of the definitions.

Lemma 3.9. If $\mathbf{C}$ is a chain in $\Pi_{\kappa}$ and $\lambda \leq \kappa$, then $(\vee \mathbf{C}) \sqcap \lambda=\vee(\mathbf{C} \sqcap \lambda)$, and $(\wedge \mathbf{C}) \sqcap \lambda=\wedge(\mathbf{C} \sqcap \lambda)$.

Proof. Let $\lambda \leq \kappa$, and $\mathbf{C}$ be a chain in $\Pi_{\kappa}$. If $\mathbf{C}$ is empty, then the result follows directly from Lemma 3.8 (i-ii) and the definition of meet and join on the empty set.

Assume now that $\mathbf{C}$ is nonempty, and fix $x \in \lambda$. For each $\mathcal{P} \in \mathbf{C}$, let $B_{\mathcal{P}}$ be the block of $\mathcal{P}$ containing $x$, and $B_{\tilde{\mathcal{P}}}$ be the block of $\tilde{\mathcal{P}}=\mathcal{P} \sqcap \lambda$ containing $x$.

Greatest lower bound. Let $B$ be the block in $\wedge \mathbf{C}$, and $B_{\lambda}$ be the block in $\wedge(\mathbf{C} \sqcap \lambda)$, that contains $x$. Applying Corollary 3.6 to both $B_{\lambda}$ and to $B$ yields

$$
B_{\lambda}=\bigcap_{\tilde{\mathcal{P}} \in \mathbf{C} \sqcap \lambda} B_{\tilde{\mathcal{P}}}=\bigcap_{\mathcal{P} \in \mathbf{C}} B_{\tilde{\mathcal{P}}}=\bigcap_{\mathcal{P} \in \mathbf{C}}\left(B_{\mathcal{P}} \cap \lambda\right)=\left(\bigcap_{\mathcal{P} \in \mathbf{C}} B_{\mathcal{P}}\right) \cap \lambda=B \cap \lambda
$$

Note that restriction is not injective, hence we may have $\mathcal{P} \neq \mathcal{P}^{\prime}$ but $\mathcal{P} \sqcap \lambda=\mathcal{P}^{\prime} \sqcap \lambda$. In this case, $B_{\tilde{\mathcal{P}}}=B_{\tilde{\mathcal{P}}^{\prime}}$, thus the second equality above is correct.

As this holds for every $x \in \lambda$, each block of $\wedge(\mathbf{C} \sqcap \lambda)$ is the restriction of a block of $\wedge \mathbf{C}$, therefore $\wedge(\mathbf{C} \sqcap \lambda)=(\wedge \mathbf{C}) \sqcap \lambda$.

Least upper bound: The proof is symmetrical with key equalities being:

$$
B_{\lambda}=\bigcup_{\tilde{\mathcal{P}} \in \mathbf{C} \sqcap \lambda} B_{\tilde{\mathcal{P}}}=\bigcup_{\mathcal{P} \in \mathbf{C}} B_{\tilde{\mathcal{P}}}=\bigcup_{\mathcal{P} \in \mathbf{C}}\left(B_{\mathcal{P}} \cap \lambda\right)=\left(\bigcup_{\mathcal{P} \in \mathbf{C}} B_{\mathcal{P}}\right) \cap \lambda=B \cap \lambda
$$

Lemma 3.10. Restriction preserves well-order and completeness of chains.

Proof. Let $\lambda \leq \kappa$ and $\mathbf{C}$ be a chain in $\Pi_{\kappa}$. By Lemma 3.8(iv), $\mathbf{C} \sqcap \lambda$ is a chain in $\Pi_{\lambda}$. Let $\mathbf{D}_{\lambda} \subseteq \mathbf{C} \sqcap \lambda$ be any, possibly empty, subset. We have $\mathbf{D}_{\lambda}=\mathbf{D} \sqcap \lambda$, with $\mathbf{D}=\left\{\mathcal{P} \in \mathbf{C} \mid \mathcal{P} \sqcap \lambda \in \mathbf{D}_{\lambda}\right\}$. Recall that subsets of chains are chains themselves, hence by Lemma 3.9. $\vee \mathbf{D}_{\lambda}=\vee(\mathbf{D} \sqcap \lambda)=(\vee \mathbf{D}) \sqcap \lambda$, and $\wedge \mathbf{D}_{\lambda}=\wedge(\mathbf{D} \sqcap \lambda)=$ $(\wedge \mathbf{D}) \sqcap \lambda$.

Well-order: If $\mathbf{C}$ is well-ordered, then $\wedge \mathbf{D} \in \mathbf{D}$ is its least element. By construction of $\mathbf{D}, \wedge \mathbf{D}_{\lambda}=(\wedge \mathbf{D}) \sqcap \lambda \in \mathbf{D}_{\lambda}$ which is thus the least element of $\mathbf{D}_{\lambda}$. Hence, $\mathbf{C} \sqcap \lambda$ is well-ordered.

Completeness: If $\mathbf{C}$ is complete, then $\wedge \mathbf{D} \in \mathbf{C}$. Hence, $\wedge \mathbf{D}_{\lambda}=(\wedge \mathbf{D}) \sqcap \lambda \in \mathbf{C} \sqcap \lambda$. Similarly, $\vee \mathbf{D}_{\lambda} \in \mathbf{C} \sqcap \lambda$. Thus, $\mathbf{C} \sqcap \lambda$ is complete.

Lemma 3.11. Restriction preserves maximality of chains.

Proof. Because of the nature of the proof, we explicitly tell in which set of partitions the inequalities hold (even when it is the whole lattice). We abusively write $\mathcal{P}<_{\lambda} \mathcal{Q}$ (and so on) instead of $\mathcal{P}<_{\Pi_{\lambda}} \mathcal{Q}$, for the sake of clarity.

Let $\lambda \leq \kappa$ and $\mathbf{C}$ be a maximal chain in $\Pi_{\kappa}$. It is complete and covering by Lemma 3.3 . Hence, $\mathbf{C} \sqcap \lambda$ is a complete chain due to Lemma 3.10 and we only need to show that it is also covering.

Let $\tilde{\mathcal{P}}, \tilde{\mathcal{Q}} \in \mathbf{C} \sqcap \lambda$ such that $\tilde{\mathcal{P}} \prec_{\mathbf{C} \sqcap \lambda} \tilde{\mathcal{Q}}$. By construction, there exist $x, y \in \lambda$ such that $x \equiv_{\tilde{\mathcal{P}}} y$ and $x \equiv_{\tilde{\mathcal{Q}}} y$, whereby $\tilde{\mathcal{P}} \in(\mathbf{C} \sqcap \lambda)_{x, y}^{-}$and $\tilde{\mathcal{Q}} \in(\mathbf{C} \sqcap \lambda)_{x, y}^{+}$. Note that because both $x$ and $y$ are in $\lambda$, we have $(\mathbf{C} \sqcap \lambda)_{x, y}^{-}=\mathbf{C}_{x, y}^{-} \sqcap \lambda$ and $(\mathbf{C} \sqcap \lambda)_{x, y}^{+}=\mathbf{C}_{x, y}^{+} \sqcap \lambda$.

By definition of restrictions, there exist (non-unique) $\mathcal{P}, \mathcal{Q} \in \mathbf{C}$ such that $\tilde{\mathcal{P}}=$ $P \sqcap \lambda$ and $\tilde{\mathcal{Q}}=\mathcal{Q} \sqcap \lambda$, and we must have $x \neq_{\mathcal{P}} y$ and $x \equiv_{\mathcal{Q}} y$, whereby $\mathcal{P} \in$ $\mathbf{C}_{x, y}^{-}$and $\mathcal{Q} \in \mathbf{C}_{x, y}^{+}$. Since $\mathbf{C}$ is maximal, it is complete and covering, hence 
Lemma $3.5(3)$ (c) yields $\vee \mathbf{C}_{x, y}^{-} \prec_{\kappa} \wedge \mathbf{C}_{x, y}^{+}$. Because they differ on $x, y \in \lambda$, we have $\left(\vee \mathbf{C}_{x, y}^{-}\right) \sqcap \lambda \prec_{\lambda}\left(\wedge \mathbf{C}_{x, y}^{+}\right) \sqcap \lambda$ by Lemma 3.8(vi), hence Lemma 3.9 yields $\vee\left(\mathbf{C}_{x, y}^{-} \sqcap \lambda\right) \prec_{\lambda} \wedge\left(\mathbf{C}_{x, y}^{+} \sqcap \lambda\right)$, i.e., $\vee(\mathbf{C} \sqcap \lambda)_{x, y}^{-} \prec_{\lambda} \wedge(\mathbf{C} \sqcap \lambda)_{x, y}^{+}$.

By completeness of $\mathbf{C} \sqcap \lambda$, it contains both $\vee(\mathbf{C} \sqcap \lambda)_{x, y}^{-}$and $\wedge(\mathbf{C} \sqcap \lambda)_{x, y}^{+}$, thus $\vee(\mathbf{C} \sqcap \lambda)_{x, y}^{-} \prec_{\mathbf{C} \sqcap \lambda} \wedge(\mathbf{C} \sqcap \lambda)_{x, y}^{+}$. Because $\tilde{\mathcal{P}} \in(\mathbf{C} \sqcap \lambda)_{x, y}^{-}$, we have $\tilde{\mathcal{P}} \leq_{\lambda} \vee(\mathbf{C} \sqcap \lambda)_{x, y}^{-}$ and similarly, $\wedge(\mathbf{C} \sqcap \lambda)_{x, y}^{+} \leq_{\lambda} \tilde{\mathcal{Q}}$,

Because $\tilde{\mathcal{P}} \prec_{\mathbf{C} \sqcap \lambda} \tilde{\mathcal{Q}}$ by definition, we must have $\tilde{\mathcal{P}}=\vee(\mathbf{C} \sqcap \lambda)_{x, y}^{-} \prec_{\mathbf{C} \sqcap \lambda} \wedge(\mathbf{C} \sqcap$ $\lambda)_{x, y}^{+}=\tilde{\mathcal{Q}}$, therefore $\tilde{\mathcal{P}} \prec_{\lambda} \tilde{\mathcal{Q}}$ and $\mathbf{C} \sqcap \lambda$ is covering. Being both complete and covering, $\mathbf{C} \sqcap \lambda$ is maximal.

\section{WELL-ORDERED CHAINS IN $\Pi_{\kappa}$}

For finite $\kappa=n$, it is immediate that any maximal chain in $\Pi_{n}$ has cardinality $n$ : Each step reduces the number of blocks by 1, whereby going from $\perp$ with $n$ blocks to $T$ with one block requires $n-1$ steps, hence $n$ elements in the chain. For $n \geq 3$, maximal chains are not unique. In this section, we show that maximal well-ordered chains in $\Pi_{\kappa}$ always have cardinality $\kappa$, whether $\kappa$ is finite or infinite.

If a maximal chain in $\Pi_{\kappa}$ is well-ordered of order type $\alpha$, then $\alpha$ is a successor ordinal ${ }^{1}$ For clarity, we will write the order type of a maximal chain as $\alpha+1$ to emphasize that it is a successor ordinal. Because $|\alpha|=|\alpha+1|$, this has no impact on the cardinality of the chain. Such chains can be written as $\mathbf{C}=\left\{\mathcal{P}_{\beta} \mid \beta \leq \alpha\right\}$ - or as $\mathbf{C}=\left\{\mathcal{P}_{\beta} \mid \beta<\alpha+1\right\}$ to emphasize the order type — with $\mathcal{P}_{0}=\perp$ and $\mathcal{P}_{\alpha}=\top$.

Lemma 4.1. Let $\kappa$ be any cardinal. If a chain in $\Pi_{\kappa}$ is well-ordered of order type $\alpha$, then $|\alpha| \leq \kappa$.

Note that we are here speaking of any well-ordered chain, not necessarily a maximal one, so its order type may be anything.

Proof. The lemma holds trivially when $\kappa$ is finite, as detailed above. Assume now that $\kappa$ is an infinite cardinal. As $\Pi_{\kappa}$ is isomorphic to $\operatorname{Equ}(\kappa)$, the set of equivalence relations on $\kappa$ ordered by $\subseteq$, there is, for any chain of order type $\alpha$ in $\Pi_{\kappa}$, a chain of order type $\alpha$ in the poset $(\mathscr{P}(\kappa \times \kappa), \subseteq)$. Let $\mathbf{C}=\left\{\mathcal{E}_{\beta} \mid \beta<\alpha\right\}$ be such a chain and observe that for every $\beta$ with $\beta+1<\alpha$ there exists at least one $\gamma_{\beta} \in \mathcal{E}_{\beta+1} \backslash \mathcal{E}_{\beta}$. As $\mathbf{C}$ is totally ordered under $\subseteq$, this implies $\gamma_{\beta} \notin \mathcal{E}_{\beta^{\prime}}$ when $\beta^{\prime}<\beta$, i.e. the $\gamma_{\beta}$ do not repeat. Hence, $\left\{\gamma_{\beta} \mid \beta+1<\alpha\right\} \subseteq \kappa \times \kappa$ has cardinality $|\alpha|$, implying $|\alpha| \leq|\kappa \times \kappa|=\kappa$.

Lemma 4.2. Every well-ordered maximal chain of order type $\alpha+1$ in $\Pi_{\kappa}$ satisfies $\operatorname{cf}(\kappa) \leq|\alpha|$.

Proof. Let $\mathbf{C}=\left\{\mathcal{P}_{\beta} \mid \beta<\alpha+1\right\}$ be a maximal well-ordered chain in $\Pi_{\kappa}$ of order type $\alpha+1$. Consider the partitions in this chain that have at least one block of cardinality $\kappa$. Since $T=\mathcal{P}_{\alpha}$, there is at least one such partition. Let $\delta$ be the least ordinal such that $\mathcal{P}_{\delta}$ contains a block of cardinality $\kappa$. It exists, because every non-empty set of ordinals has a least element. Let $B^{\delta}$ be a block of cardinality $\kappa$ in $\mathcal{P}_{\delta}$.

By Lemma 3.2 and maximality of $\mathbf{C}$ we have $\vee \mathbf{C}_{\mathcal{P}_{\delta}}^{-} \preceq \mathcal{P}_{\delta}$, and $\vee \mathbf{C}_{\mathcal{P}_{\delta}}^{-} \in \mathbf{C}$. That is, we can write $\mathcal{P}_{\gamma}=\vee \mathbf{C}_{\mathcal{P}_{\delta}}^{-}$either for $\gamma=\delta$ or $\gamma+1=\delta$. Consider for the sake of contradiction that $\mathcal{P}_{\gamma} \prec \mathcal{P}_{\delta}$. Then $B^{\delta}$ would be either a block of $\mathcal{P}_{\gamma}$ or the union

\footnotetext{
${ }^{1}$ Any maximal chain in $\Pi_{\kappa}$ has a maximal element, namely $\top$. But since a well-ordered set of limit-ordinal type has no maximal element, this implies that the order type of a well-ordered maximal chain must be a successor-ordinal.
} 
of two such blocks, at least one of them with cardinality $\kappa$. Both cases contradict the hypothesis of $\delta$ being the smallest ordinal for which $\mathcal{P}_{\delta}$ contains a block of cardinality $\kappa$. Hence $\delta=\gamma$, and $\mathcal{P}_{\delta}=\vee \mathbf{C}_{\mathcal{P}_{\delta}}^{-}=\vee_{\beta<\delta} \mathcal{P}_{\beta}$.

Corollary 3.6 implies that a sequence $\left\{B^{\beta}\right\}_{\beta<\delta}$ exists such that $B^{\delta}=\bigcup_{\beta<\delta} B^{\beta}$. By definition of $\delta$, we have $\left|B^{\beta}\right|<\kappa$ for every $\beta<\delta$, and so by the definition of co-finality, $\operatorname{cf}(\kappa) \leq|\delta| \leq|\alpha|$

Corollary 4.3. If $\kappa$ is a regular cardinal, then every well-ordered maximal chain in $\Pi_{\kappa}$ indexed by an ordinal $\alpha$ satisfies $|\alpha|=\kappa$.

Theorem 4.4. If $\kappa$ is any infinite cardinal, then every well-ordered maximal chain of order type $\alpha+1$ in $\Pi_{\kappa}$ satisfies $|\alpha|=\kappa$.

Proof. Because of the previous results, we only need to check that $|\alpha| \geq \kappa$ whenever $\kappa$ is singular. Indeed, the case of regular $\kappa$ is handled by Corollary 4.3 and the upper bound is due to Lemma 4.1 .

Cardinal arithmetic: It suffices to show that $\lambda \leq|\alpha|$ for every regular $\lambda<\kappa$ : By standard cardinal arithmetic, the singular $\kappa$ can be expressed as the sum of $\operatorname{cf}(\kappa)$ smaller successor (hence regular) cardinals $\lambda_{\delta}$. If the above inequality holds, then:

$$
\kappa=\sum_{\delta \leq \operatorname{cf}(\kappa)} \lambda_{\delta} \leq \sum_{\delta \leq \operatorname{cf}(\kappa)}|\alpha|=\operatorname{cf}(\kappa) \cdot|\alpha|=\max (\operatorname{cf}(\kappa),|\alpha|)
$$

And because $\kappa>\operatorname{cf}(\kappa)$ by singularity, we can finally conclude that $\kappa \leq|\alpha|$.

Cardinality: Let $\mathbf{C}$ be a maximal, well-ordered chain in $\Pi_{\kappa}$. By Lemmas 3.10 and 3.11, $\mathbf{C} \sqcap \lambda$ is a maximal well-ordered chain in $\Pi_{\lambda}$ for every regular $\lambda<\kappa$. Hence, by Corollary 4.3 , we have $|\mathbf{C} \sqcap \lambda|=\lambda$. Moreover, by construction, $|\mathbf{C} \sqcap \lambda| \leq$ $|\mathbf{C}|=|\alpha|$. Thus, for any regular $\lambda<\kappa$ we have $\lambda \leq|\alpha|$ and we can conclude that $\kappa \leq|\alpha|$, whereby $\kappa=|\alpha|$.

Remark 4.5. Notice that any ordinal $\alpha+1$ with $|\alpha|=\kappa$ appears as the order type of some maximal well-ordered chain in $\Pi_{\kappa}$. Indeed, the chain of singular partitions in $\Pi(\alpha+1)$ with non-singleton block $B_{\beta}=\{\delta \mid \delta \leq \beta\}$ works. In other words, it suffices to find a well-order of order type $\alpha+1$ on $\kappa$ (which exists by a cardinality argument) and add the elements to a single block in this order.

\section{LONG CHAINS IN $\Pi_{\kappa}$}

For any nonempty $S \subseteq \kappa$, we define the partition

$$
\operatorname{diag}(S)=\{S\} \cup\{\{\gamma\} \mid \gamma \in \kappa \backslash S\} .
$$

When $|S| \geq 2, \operatorname{diag}(S)$ is the singular partition with non-singular block $S$.

Remark 5.1. It is easy to verify that $\operatorname{diag}(-)$ is an order isomorphism between subsets of $\kappa$ of size $\geq 2$ and the singular partitions. In particular, chains in $(\mathscr{P}(\kappa), \subseteq$ ) that contain only sets of length $\geq 2$ are mapped to chains with the same cardinality in $\left(\Pi_{\kappa}, \leq\right)$.

Theorem 5.2. Let $\kappa$ be an infinite cardinal. There is a chain of cardinality $>\kappa$ in $\Pi_{\kappa}$. The chain may be chosen to be maximal.

Proof. By a result of Sierpiński Sie22] (see also [Har05, Thm. 4.7.35]), there is a chain $\mathbf{D}^{\prime}$ in the poset $(\mathscr{P}(\kappa), \subseteq)$ of cardinality $\lambda>\kappa$. There is at most one singleton element $\{\alpha\} \in \mathbf{D}^{\prime}$, and as $\lambda$ is infinite, $\mathbf{D}=\mathbf{D}^{\prime} \backslash\{\emptyset,\{\alpha\}\}$ is still a chain of cardinality $\lambda$.

Then, by Remark 5.1 the set $\mathbf{C}=\{\operatorname{diag}(S) \mid S \in \mathbf{D}\}$ is a chain in $\Pi_{\kappa}$ of cardinality $\lambda>\kappa$. By the Maximal Chain Theorem, any chain in a poset is contained in a maximal chain, and the result follows. 
We note that Theorem 5.2 shows that under GCH, there are chains whose cardinalities reach the trivial upper bound, $2^{\kappa}$. For the special case of $\kappa=\aleph_{0}$, we may obtain existence of a maximal chain in $\Pi_{\aleph_{0}}$ of cardinality $2^{\aleph_{0}}$ without use of (G) $\mathrm{CH}$ :

Lemma 5.3. There is a maximal chain of cardinality $2^{\aleph_{0}}$ in $\Pi_{\aleph_{0}}$.

Proof. For each $r \in \mathbb{R}$, define the left Dedekind cut $D_{r}=\{q \in \mathbb{Q} \mid q<r\}$ and note that $r<r^{\prime}$ implies $D_{r} \subsetneq D_{r^{\prime}}$ by density of $\mathbb{Q}$ in $\mathbb{R}$. Hence, the set of left Dedekind cuts is a chain in $\mathscr{P}(\mathbb{Q})$ of cardinality $|\mathbb{R}|=2^{\aleph_{0}}$, all elements of which have cardinality $\geq 2$, and consequently, by Lemma 5.1 , the set of partitions $\left\{\operatorname{diag}\left(D_{r}\right) \mid r \in \mathbb{R}\right\}$ is a chain in $\Pi_{\aleph_{0}}$ of cardinality $2^{\aleph_{0}}$. By the Maximal Chain Theorem, this chain can be extended to a maximal chain; note that $2^{\aleph_{0}}$ is an upper bound on the cardinality of any chain in $\Pi_{\aleph_{0}}$.

For arbitrary infinite $\kappa$ we do not know whether existence of a maximal chain in $\Pi_{\kappa}$ of cardinality $2^{\kappa}$ can be proved without assuming $\mathrm{GCH}$.

\section{Short Chains}

We now turn to the question of whether there are maximal chains of cardinality strictly less than $\kappa$ in $\Pi_{\kappa}$. It is immediate that there are no maximal chains of finite cardinality in $\Pi_{\aleph_{0}}$. Indeed, each step in a maximal chain merges exactly two blocks. Hence, starting from $\perp$ which has infinitely many blocks, after a finite number of steps it is impossible to reach $T$ or any other partition that has only finitely many blocks. For larger cardinals, there is a general construction that proves existence of short chains. In the special case where $\mathrm{GCH}$ is assumed, it proves existence of a maximal chain of cardinality $\kappa^{-}$for every successor cardinal $\kappa$. Because this construction is notationally cumbersome, we first explain the result for the particular case of $\kappa=2^{\aleph_{0}}$, the cardinality of the continuum, in order to aid the reader's understanding.

\subsection{Short chains in $\Pi_{2^{\aleph_{0}}}$.}

We build a maximal chain of size $\aleph_{0}$ in $\Pi_{2 \aleph_{0}}$. The proof boils down to the fact that there are countably many binary strings of finite length but uncountably many binary strings of countably infinite length, hence an infinite binary tree of depth $\omega$ has uncountably many leaves but only countably many inner nodes.

The construction proceeds in two steps:

(1) Starting from $T$, inductively construct a countable chain of "keyframe" partitions such that the $n^{\text {th }}$ keyframe has $2^{n}$ blocks. The greatest lower bound of this chain will be $\perp$ with $2^{\aleph_{0}}$ singleton blocks.

(2) Complete the chain into a maximal chain by adding "inbetween" partitions between the keyframes. We will need $2^{n}-1$ extra partitions between the $(n-1)^{\text {st }}$ and the $n^{\text {th }}$ keyframe $2^{2}$

As a finite number of inbetween partitions are added for each $n$, only a countable number of partitions are added in total, and so the full chain has countable length.

Keyframes. Consider partitions of the set $\{0,1\}^{\omega}$ of countably infinite sequences of bits; note that the cardinality of $\{0,1\}^{\omega}$ is $2^{\aleph_{0}}$. Let $u \in\{0,1\}^{\omega}$ and let $[u]_{k}$ be the set of elements of $\{0,1\}^{\omega}$ with the same first $k$ bits: $[u]_{k}=$ $\left\{u_{0} u_{1} \ldots u_{k-1} v \mid v \in\{0,1\}^{\omega}\right\}$. Define the $k^{\text {th }}$ keyframe to be $\mathcal{K}_{k}=\left\{[u]_{k} \mid u \in\{0,1\}^{\omega}\right\}$. Similarly, define $\mathcal{K}_{\omega}=\perp$.

\footnotetext{
2 "Keyframe" and "Inbetween" are terms from animation: A "keyframe" is a drawing that defines the start or end of a movement. The animation frames between keyframes-the "inbetweens"are drawn to make the transitions between keyframes smooth.
} 


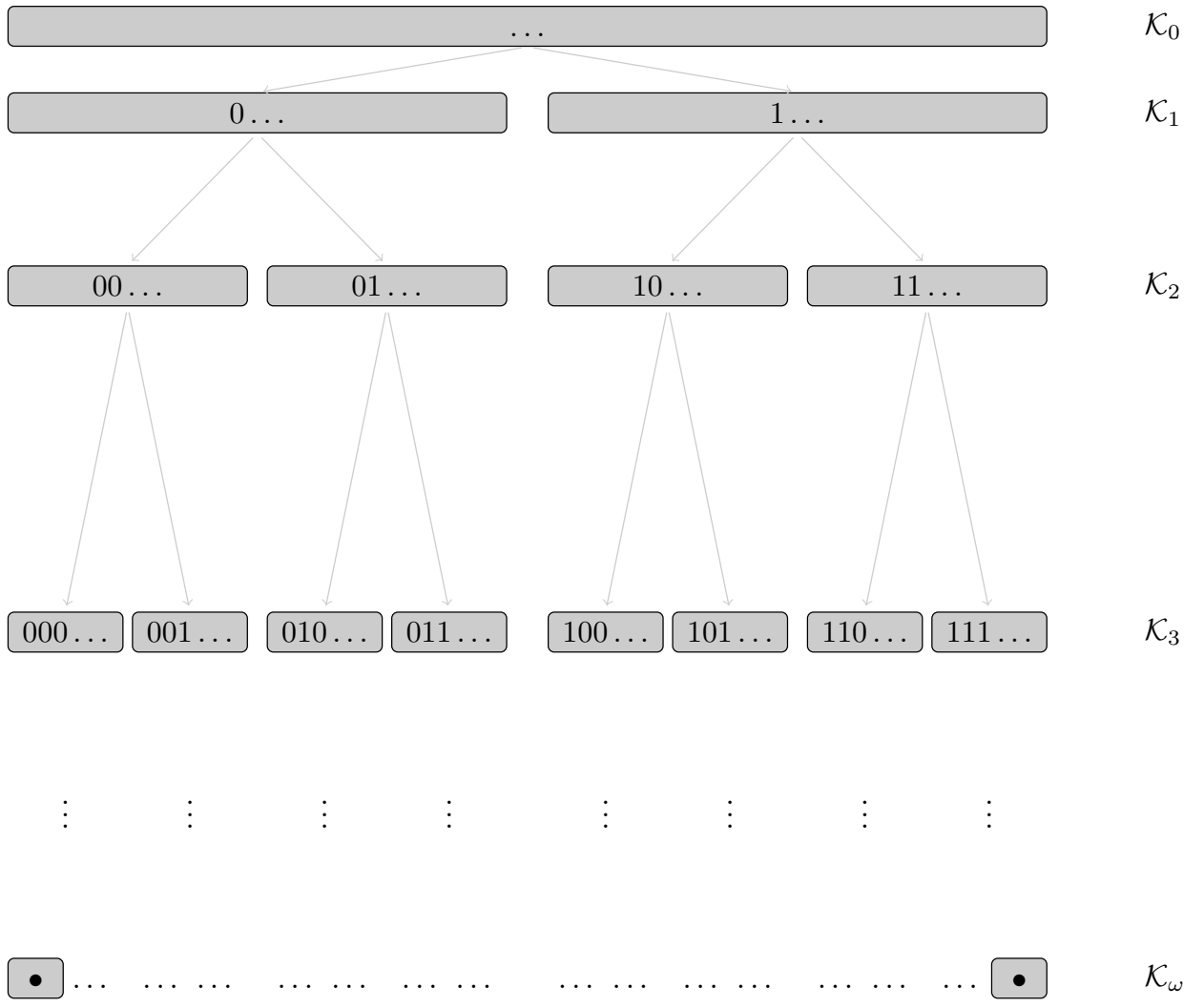

FIGURE 1. Keyframe partitions

Thus, the keyframes are as shown in Figure 1. Each level of the picture corresponds to one partition, with its blocks depicted. It is instructive to note that the set of blocks of all partitions form an infinite binary tree and that the number of nodes in each keyframe is finite (whence the corresponding partition has only finitely many blocks). The blocks of $\mathcal{K}_{\omega}$ constitute the bottom most level of the tree, and hence are the leaves of the infinite binary tree, of which there are $2^{|\omega|}$. The total number of keyframe partitions corresponds to the number of levels in the infinite tree, and is thus clearly countable. The total number of internal nodes in the tree is $\sum_{\alpha<\omega} 2^{|\alpha|}=\aleph_{0}$.

Completing the chain. The set of keyframe partitions clearly form a chain, but just as clearly this chain is not maximal. Inbetween partitions must be added between the keyframes to obtain a maximal chain.

As seen in Figure 1, between the $k^{\text {th }}$ and the $(k+1)^{\text {st }}$ keyframe, $2^{k}$ blocks have been split. In order to locally saturate the chain, it suffices to add $2^{k}-1$ new partitions, each with one more of the $2^{k}$ blocks split in two. This will ensure that each partition is a successor to the previous one.

After adding these new partitions, the picture is now as shown in Figure 2. This chain is easily seen to be maximal. At each (non-keyframe) partition, the new blocks, resulting from splitting one of the blocks of the parent partition, are shown with hatching.

Since there are $2^{k}$ inbetween partitions between the $k^{\text {th }}$ and $(k+1)^{\text {st }}$ keyframes, the total number of inbetween partitions is $\sum_{k<\omega} 2^{k}$, which is countable. Since there are also only countably many keyframes, the total number of partitions in the chain is countable. 


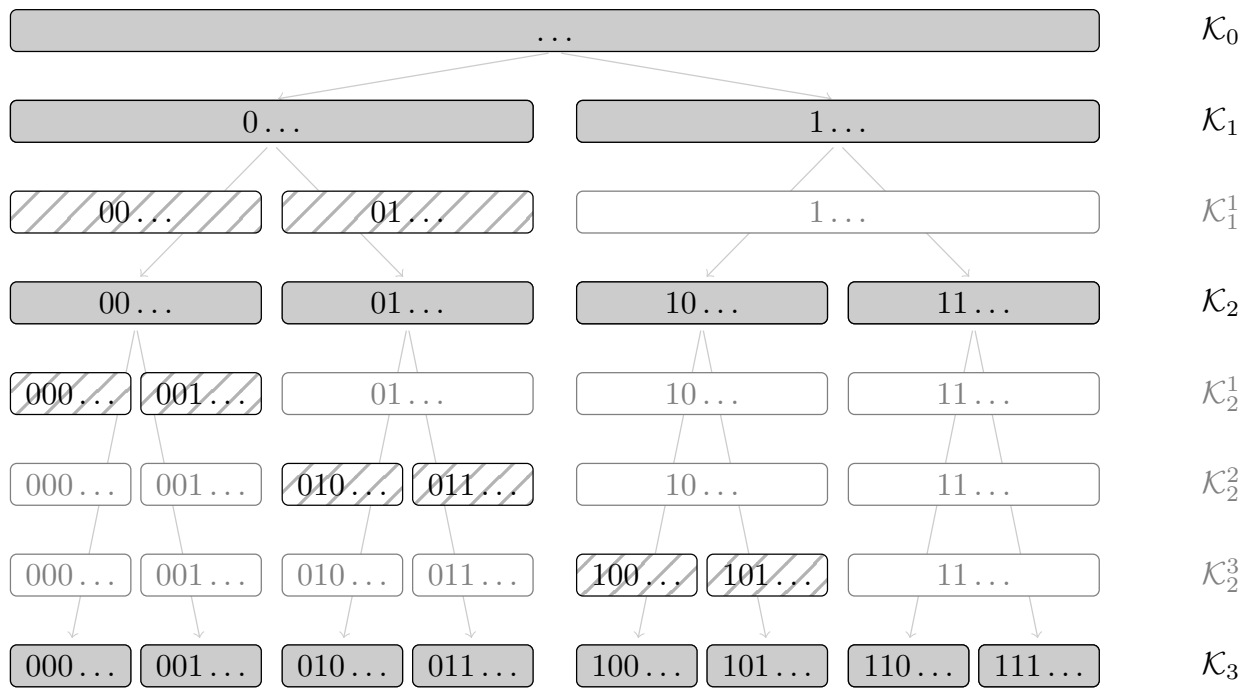

Figure 2. A short maximal chain

6.2. Short chains: general construction for large cardinals. Let $\lambda$ be any infinite cardinal and $\kappa=2^{\lambda}$. We will build a maximal chain of cardinality strictly less than $\kappa$ in $\Pi_{\kappa}=\Pi_{2^{\lambda}}$.

Underlying set. Let $S$ be the set of binary sequences indexed by the initial ordinal of $\lambda$. That is $S=\{0,1\}^{\omega_{\lambda}}$. $S$ contains $\kappa$ elements; we shall use $\Pi(S)$ as a concrete instance of $\Pi_{\kappa}$.

Keyframes. Let $f \in S$ and $\delta \leq \omega_{\lambda}$. We denote by $[f]_{\delta}$ the set of sequences that agree with $f$ on the initial $\delta$ elements:

$$
[f]_{\delta}=\{g \in S \mid \forall \beta<\delta, f(\beta)=g(\beta)\}
$$

Observe that $[f]_{\delta}$ is uniquely defined by a binary sequence indexed by $\delta$. The bracketed notation $[f]$ is used to emphasize that $[f]$ is truly the equivalence class of $f$ in the corresponding equivalence, even though everything is written in terms of partitions.

Let $\mathcal{K}_{\delta}=\left\{[f]_{\delta} \mid f \in S\right\}$. Then, $\mathcal{K}_{\delta}$ is a partition of $S$ which we call a keyframe partition. From the definition, it follows that $\mathcal{K}_{0}=\top$ and that $\mathcal{K}_{\omega_{\lambda}}=\perp$. Note that $\mathcal{K}_{\delta}$ consists of $2^{|\delta|}$ blocks and is in bijective correspondence with $\{0,1\}^{\delta}$. Let $\mathbf{K}=\left\{\mathcal{K}_{\delta} \mid \delta \leq \omega_{\lambda}\right\}$

Lemma 6.1. If $\alpha<\beta$ then $[f]_{\beta} \subset[f]_{\alpha}$ and $\mathcal{K}_{\beta}<\mathcal{K}_{\alpha}$. Furthermore, $\mathbf{K}$ is a chain in $\Pi_{\kappa}$.

Proof. The first point is immediate by definition of the refinement ordering: if $f$ and $g$ agree on their initial $\beta$ elements, then they trivially agree on their initial $\alpha$ elements, so $[f]_{\beta} \subsetneq[f]_{\alpha}$. Since this holds for every $f \in S$, we further get $\mathcal{K}_{\beta}<\mathcal{K}_{\alpha}$. The second point is an immediate consequence of the first. 
Lemma 6.2. $\mathbf{K}$ is closed.

Proof. Let $D$ be a set of ordinals and $\mathbf{D}=\left\{\mathcal{K}_{\delta} \mid \delta \in D\right\}$. Being a set of ordinals, $D$ admits a minimal element $\alpha$. Then $\mathcal{K}_{\alpha}$ is an upper bound for $\mathbf{D}$ and being part of it, must be the least upper bound.

Let $\beta$ be the smallest ordinal not in $D$. If $\beta=\beta^{\prime}+1$ is a successor ordinal, then $\mathcal{K}_{\beta^{\prime}}$ is both part of $\mathbf{D}$ and a lower bound, hence it's the greatest lower bound.

Suppose that $\beta$ is a limit ordinal and that $\wedge \mathbf{D}>\mathcal{K}_{\beta}$. Then, there exists $f, g$ such that $f \equiv \wedge \mathbf{D} g$ but $f \not \equiv_{\mathcal{K}_{\beta}} g$. By definition of $\mathcal{K}_{\beta}$, there exists $\gamma<\beta$ with $f(\gamma) \neq g(\gamma)$. However, by definition of $\beta$, there must exists $\gamma^{\prime} \in D$ with $\gamma \leq \gamma^{\prime}<\beta$. $f(\gamma) \neq g(\gamma)$ thus implies $f \not \equiv_{\mathcal{K}_{\gamma^{\prime}}} g$ which contradicts $f \equiv \wedge \mathbf{D} g$. Therefore, $\mathbf{K}$ is closed.

Locally saturating the chain. We fix $\delta<\omega_{\lambda}$ and construct a saturated chain bounded below by $\mathcal{K}_{\delta+1}$ and above by $\mathcal{K}_{\delta}$.

$\mathcal{K}_{\delta}$ has cardinality $2^{|\delta|}$. Fix, by the Axiom of Choice, a well-ordering $<_{\delta}$ of $\mathcal{K}_{\delta}$ with order type $\omega_{2^{|\delta|}}$. For $0 \leq \alpha<\omega_{2^{|\delta|}}$, let the block $S_{\delta, \alpha}$ be the $\alpha^{\text {th }}$ element of $\mathcal{K}_{\delta}$ according to this ordering.

For each $0 \leq \alpha<\omega_{2^{|\delta|}}$, define the set $[f]_{\delta}^{\alpha}$ as

$$
[f]_{\delta}^{\alpha}= \begin{cases}{[f]_{\delta+1}} & \text { if }[f]_{\delta}<_{\delta} S_{\delta, \alpha} \\ {[f]_{\delta}} & \text { if } S_{\delta, \alpha} \leq_{\delta}[f]_{\delta}\end{cases}
$$

and let $\mathcal{K}_{\delta}^{\alpha}=\left\{[f]_{\delta}^{\alpha} \mid f \in S\right\}$. Observe that the sets $[f]_{\delta}^{\alpha}$ are pairwise disjoint and that their union is $S$. Thus, $\mathcal{K}_{\delta}^{\alpha}$ is a partition of $S$ (an "inbetween" partition), obtained by splitting the initial $\alpha$ blocks of $\mathcal{K}_{\delta}$. By construction there are $2^{|\delta|}$ inbetween partitions between $\mathcal{K}_{\delta}$ and $\mathcal{K}_{\delta+1}$.

Lemma 6.3. For all $f \in S,[f]_{\delta}^{0}=[f]_{\delta}$. Thus $\mathcal{K}_{\delta}^{0}=\mathcal{K}_{\delta}$.

Proof. By construction, $S_{\delta, 0}$ is minimal for $<_{\delta}$, thus it is impossible for $[f]_{\delta}$ to be strictly smaller.

Lemma 6.4. Let $\alpha<\beta$. Then for each $f,[f]_{\delta}^{\beta} \subseteq[f]_{\delta}^{\alpha}$.

Proof. By cases depending on $<_{\delta}$. First note that $\alpha<\beta$ implies $S_{\delta, \alpha}<_{\delta} S_{\delta, \beta}$ by construction.

(1) If $[f]_{\delta}<{ }_{\delta} S_{\delta, \alpha}<{ }_{\delta} S_{\delta, \beta}$ then $[f]_{\delta}^{\beta}=[f]_{\delta+1}=[f]_{\delta}^{\alpha}$.

(2) If $S_{\delta, \alpha} \leq_{\delta}[f]_{\delta}<_{\delta} S_{\delta, \beta}$ then $[f]_{\delta}^{\beta}=[f]_{\delta+1} \subsetneq[f]_{\delta}=[f]_{\delta}^{\alpha}$.

(3) If $S_{\delta, \alpha}<_{\delta} S_{\delta, \beta} \leq_{\delta}[f]_{\delta}$ then $[f]_{\delta}^{\beta}=[f]_{\delta}=[f]_{\delta}^{\alpha}$.

Lemma 6.5. Let $\alpha<\beta$. Then $\mathcal{K}_{\delta}^{\beta}<\mathcal{K}_{\delta}^{\alpha}$.

Proof. By Lemma 6.4 each block of $\mathcal{K}_{\delta}^{\beta}$ is contained in a block of $\mathcal{K}_{\delta}^{\alpha}$, thus $\mathcal{K}_{\delta}^{\beta} \leq \mathcal{K}_{\delta}^{\alpha}$.

Since $\alpha<\beta$, there exists $f$ such that $S_{\delta, \alpha} \leq_{\delta}[f]_{\delta}<_{\delta} S_{\delta, \beta}$. Hence there are blocks of $\mathcal{K}_{\delta}^{\beta}$ that are strictly contained in blocks of $\mathcal{K}_{\delta}^{\alpha}$, and thus the inequality is indeed strict.

Let $\mathbf{C}_{\delta}^{\prime}=\left\{\mathcal{K}_{\delta}^{\alpha} \mid 0 \leq \alpha<\omega_{2^{|\delta|}}\right\}$ and $\mathbf{C}_{\delta}=\mathbf{C}_{\delta}^{\prime} \cup\left\{\mathcal{K}_{\delta+1}\right\}$.

Corollary 6.6. $\mathbf{C}_{\delta}$ is a chain with minimum $\mathcal{K}_{\delta+1}$ and maximum $\mathcal{K}_{\delta}=\mathcal{K}_{\delta}^{0}$.

Note that including $\mathcal{K}_{\delta+1}$ in $\mathbf{C}_{\delta}^{\prime}$ (that is, defining only $\mathbf{C}_{\delta}$ ) appears to be more natural, but because it is both an element of $\mathbf{C}_{\delta}$ and of $\mathbf{C}_{\delta+1}$, it becomes notationally awkward when taking unions of chains and computing the cardinality of such unions.

Lemma 6.7. $\mathbf{C}_{\delta}$ is closed. 
Proof. Let $D$ be a set of ordinals and $\mathbf{D}=\left\{\mathcal{K}_{\delta}^{\alpha} \mid \alpha \in D\right\}$. Being a set of ordinals $D$ admits a minimal element $\alpha^{\prime}$. Then $\mathcal{K}_{\delta}^{\alpha^{\prime}}$ is an upper bound for $\mathbf{D}$ and being part of it, must be the least upper bound.

Let $\beta$ be the smallest ordinal not in $D$. If $\beta=\beta^{\prime}+1$ is a successor ordinal, then $\mathcal{K}_{\delta}^{\beta^{\prime}}$ is both part of $\mathbf{D}$ and a lower bound, hence it's the greatest lower bound.

Suppose that $\beta$ is a limit ordinal and that $\mathcal{K}_{\delta+1} \leq \mathcal{K}_{\delta}^{\beta}<\wedge \mathbf{D} \leq \mathcal{K}_{\delta}$. Then, there exists $f, g$ such that $f \not \equiv_{\mathcal{K}_{\delta}^{\beta}} g$ but $f \equiv_{\wedge \mathbf{D}} g$ hence $f \equiv_{\mathcal{K}_{\delta}} g$. The later implies $[f]_{\delta}=[g]_{\delta}$, therefore we must have $[f]_{\delta}<_{\delta} S_{\delta, \beta}$ in order to have $[f]_{\delta}^{\beta}=[f]_{\delta+1} \neq$ $[g]_{\delta+1}=[g]_{\delta}^{\beta}$.

Now, because $\beta$ is limit, there exists $\gamma$ with $[f]_{\delta}<_{\delta} S_{\delta, \gamma}<S_{\delta, \beta}$ and by definition of $\beta$, we can choose $\gamma \in D$. However, this implies $[f]_{\delta}^{\gamma}=[f]_{\delta+1} \neq[g]_{\delta+1}=[g]_{\delta}^{\gamma}$ and thus $f \not \equiv_{\mathcal{K}_{\delta}^{\gamma}} g$, contradicting $f \equiv \wedge \mathbf{D} g$. Therefore, $\mathbf{C}_{\delta}$ is closed.

For any $f \in S$ let $f_{0} \in[f]_{\delta}$ (resp. $f_{1} \in[f]_{\delta}$ ) be the binary sequence such that $f_{0}\left(\delta^{\prime}\right)=0$ (resp. $f_{1}\left(\delta^{\prime}\right)=1$ ) for all $\delta^{\prime} \geq \delta$. Notice that $\left[f_{0}\right]_{\delta}=\left[f_{1}\right]_{\delta}$ but $\left[f_{0}\right]_{\delta+1} \neq\left[f_{1}\right]_{\delta+1}$.

For any $g \in[f]_{\delta}$, either $g(\delta)=0$ and $[g]_{\delta+1}=\left[f_{0}\right]_{\delta+1}$, or $g(\delta)=1$ and $[g]_{\delta+1}=$ $\left[f_{1}\right]_{\delta+1}$. Thus, $[f]_{\delta}=\left[f_{0}\right]_{\delta+1} \cup\left[f_{1}\right]_{\delta+1}$.

Lemma 6.8. For every $\alpha<\omega_{2^{|\delta|}}, \mathcal{K}_{\delta}^{\alpha+1} \prec \mathcal{K}_{\delta}^{\alpha}$.

Proof. Fix $\delta$ and $\alpha$. Let $f \in S$ and consider the block $[f]_{\delta}^{\alpha}$ of $\mathcal{K}_{\delta}^{\alpha}$. There are three cases:

(1) If $[f]_{\delta}<{ }_{\delta} S_{\delta, \alpha}<{ }_{\delta} S_{\delta, \alpha+1}$ then $[f]_{\delta}^{\alpha}=[f]_{\delta}^{\alpha+1}=[f]_{\delta+1}$

(2) If $S_{\delta, \alpha}<_{\delta} S_{\delta, \alpha+1} \leq_{\delta}[f]_{\delta}$ then $[f]_{\delta}^{\alpha}=[f]_{\delta}=[f]_{\delta}^{\alpha+1}$.

(3) If $[f]_{\delta}=S_{\delta, \alpha}<_{\delta} S_{\delta, \alpha+1}$ then either $[f]_{\delta}^{\alpha+1}=\left[f_{0}\right]_{\delta+1}$ or $[f]_{\delta}^{\alpha+1}=\left[f_{1}\right]_{\delta+1}$.

(1) and (2) follow immediately from the definition. For case (3), we first obtain $[f]_{\delta}^{\alpha}=[f]_{\delta}$ and $[f]_{\delta}^{\alpha+1}=[f]_{\delta+1}$ by definition. But since $[f]_{\delta}=\left[f_{0}\right]_{\delta+1} \cup\left[f_{1}\right]_{\delta+1}$, we must have either $f \in\left[f_{0}\right]_{\delta+1}$ or $f \in\left[f_{1}\right]_{\delta+1}$. Hence $[f]_{\delta}^{\alpha+1}=[f]_{\delta+1}$ is either $\left[f_{0}\right]_{\delta+1}$ or $\left[f_{1}\right]_{\delta+1}$, as desired. By the above, every block of $\mathcal{K}_{\delta}^{\alpha}$ is a block of $\mathcal{K}_{\delta}^{\alpha+1}$ except for a single block (the unique block for which $[f]_{\delta}=S_{\delta, \alpha}$ ), which is obtained by taking the union of exactly two blocks of $\mathcal{K}_{\delta}^{\alpha+1}$. Hence, $\mathcal{K}_{\delta}^{\alpha+1} \prec \mathcal{K}_{\delta}^{\alpha}$.

Lemma 6.9. $\mathrm{C}_{\delta}$ is covering.

Proof. Let $\mathcal{P} \prec \mathbf{C}_{\delta} \mathcal{Q}$ be two partitions of $\mathbf{C}_{\delta}$. By definition, we must have $\mathcal{Q}=\mathcal{K}_{\delta}^{\alpha}$ for some $\alpha$, therefore $\mathcal{P}=\mathcal{K}_{\delta}^{\alpha+1}$. By the previous lemma, we know that $\mathcal{K}_{\delta}^{\alpha+1} \prec \mathcal{K}_{\delta}^{\alpha}$, hence $\mathbf{C}_{\delta}$ is covering.

Proposition 6.10. $\mathbf{C}_{\delta}$ is a saturated chain in $\Pi_{\kappa}$, with minimum $\mathcal{K}_{\delta+1}$ and maximum $\mathcal{K}_{\delta}$.

Proof. Applying previous results, we find that $\mathbf{C}_{\delta}$ is an endpoint-including (Corollary 6.6), closed (Lemma 6.7) and covering (Lemma 6.9) chain. Therefore, by Lemma 3.3 , it is saturated.

Completing the chain. In the following, let $\mathbf{C}=\{\perp\} \cup\left(\bigcup_{0 \leq \delta<\omega_{\lambda}} \mathbf{C}_{\delta}^{\prime}\right)$.

Theorem 6.11. $\mathrm{C}$ is a maximal chain in $\Pi_{\kappa}$.

Proof. By Proposition 6.10, $\mathbf{C}_{\delta}=\mathbf{C}_{\delta}^{\prime} \cup\left\{\mathcal{K}_{\delta+1}\right\}$ is a saturated chain with minimum $\mathcal{K}_{\delta+1}$ and maximum $\mathcal{K}_{\delta}$, whereby each $\mathbf{C}_{\delta}^{\prime}$ is a chain. Noting that $\delta<\gamma$ implies $\mathcal{K}_{\gamma}<\mathcal{K}_{\delta}$ by Lemma 6.1, every element of $\mathbf{C}_{\delta}^{\prime}$ is comparable to every element of $\mathbf{C}_{\gamma}^{\prime}$. Hence, $\mathbf{C}$ is a chain.

Suppose that there exists a partition $\mathcal{P} \notin \mathbf{C}$ such that $\mathbf{C} \cup\{\mathcal{P}\}$ is still a chain. We obviously have $\mathcal{P} \notin \mathbf{K} \subset \mathbf{C}$ and because $\mathbf{K}$ is closed (Lemma 6.2) we can apply 
Lemma 3.2 and conclude that $\vee \mathbf{K}_{\mathcal{P}}^{-} \prec_{\mathbf{K}} \wedge \mathbf{K}_{\mathcal{P}}^{+}$. Because they are both keyframes, we must have $\wedge \mathbf{K}_{\mathcal{P}}^{+}=\mathcal{K}_{\beta}$ for some ordinal, hence $\vee \mathbf{K}_{\mathcal{P}}^{-}=\mathcal{K}_{\beta+1}$. This entails $\mathcal{K}_{\beta+1}<\mathcal{P}<\mathcal{K}_{\beta}$, but we now by Proposition 6.10 that the chain is saturated between these two. Hence, $\mathbf{C}$ is maximal.

Length of the chain. Recall that $\mathbf{C}_{\delta}^{\prime}$ has cardinality $2^{|\delta|}$.

Lemma 6.12. C has cardinality $\sum_{\delta<\omega_{\lambda}} 2^{|\delta|}$.

Proof. $|\mathbf{C}|=\left|\bigcup_{\delta<\omega_{\lambda}} \mathbf{C}_{\delta}^{\prime} \cup\{\perp\}\right|=\left|\bigcup_{\delta<\omega_{\lambda}} \mathbf{C}_{\delta}^{\prime}\right|=\sum_{\delta<\omega_{\lambda}}\left|\mathbf{C}_{\delta}\right|=\sum_{\delta<\omega_{\lambda}} 2^{|\delta|}$

Lemma 6.13. Let $\lambda$ be a cardinal such that for any $\mu<\lambda$, we have $2^{\mu}<2^{\lambda}$. Then $\sum_{\delta<\omega_{\lambda}} 2^{|\delta|}<2^{\lambda}$.

Proof. If $\delta<\omega_{\lambda}$, then $|\delta|<\lambda$, thus by hypothesis, $2^{|\delta|}<2^{\lambda}$.

Assume now, for contradiction, that $\sum_{\delta<\omega_{\lambda}} 2^{|\delta|} \geq 2^{\lambda}$. Recall that

$$
\operatorname{cf}\left(2^{\lambda}\right)=\inf \left\{|I|\left|2^{\lambda}=\right| \bigcup_{i \in I} A_{i}|\wedge \forall i \in I,| A_{i} \mid<2^{\lambda}\right\}
$$

which is the same as $\operatorname{cf}\left(2^{\lambda}\right)=\inf \left\{|I| \mid 2^{\lambda}=\sum_{i \in I} \mu_{i} \wedge \forall i \in I, \mu_{i}<2^{\lambda}\right\}$.

It then follows that $\operatorname{cf}\left(2^{\lambda}\right) \leq\left|\omega_{\lambda}\right|=\lambda$. But by a standard consequence of König's Theorem Kön05] we always have $\operatorname{cf}\left(2^{\lambda}\right)>\lambda$ under the Axiom of Choice.

We can now prove our main result on short chains.

Theorem 6.14. Let $\lambda$ be an infinite cardinal such that for every cardinal $\mu<\lambda$ we have $2^{\mu}<2^{\lambda}$. Then there exists a maximal chain of cardinality $<2^{\lambda}$ (but $\geq \lambda$ ) in $\Pi_{2^{\lambda}}$.

Proof. By Theorem 6.11 and Lemma 6.12 there is a maximal chain in $\Pi_{2 \lambda}$ of cardinality $\sum_{\delta<\omega_{\lambda}} 2^{|\delta|}$. By Lemma 6.13 we have $\sum_{\delta<\omega_{\lambda}} 2^{|\delta|}<2^{\lambda}$.

Consequently, the chain is of cardinality $<2^{\lambda}$ (but $\geq \lambda$ ).

For the case of $\lambda=\aleph_{0}$, we trivially have $2^{n}<2^{\aleph_{0}}$ for all $n<\aleph_{0}$, and thus there is a countable maximal chain in $\Pi_{2^{\aleph_{0}}}$. The condition is also satisfied for all strong limit cardinals.

\section{ANTICHAINS AND MAXIMAL ANTICHAINS}

An antichain in $(\mathbf{P}, \leq)$ is a subset $\mathbf{A} \subseteq \mathbf{P}$ in which no two distinct elements of $\mathbf{P}$ are $\leq$-comparable. An antichain is maximal if adding an element to it results in a set that is not an antichain. Observe that in a poset, the trivial antichains $\{\perp\}$ and $\{\top\}$ are always maximal antichains.

There is no known tight bound on the cardinality of maximal antichains in $\Pi_{n}$ for finite $n$, but some asymptotic results are known [Can98, BH02]. The cardinality of a maximal antichain in $\Pi_{n}$ is $\Theta\left(n^{a}(\log n)^{-a-1 / 4} S\left(n, K_{n}\right)\right)$ where $a=(2-e \log 2) / 4$ and $S\left(n, K_{n}\right)=\max _{k}\left\{\begin{array}{l}n \\ k\end{array}\right\}$ is the largest Stirling number of the second kind for fixed $n$.

Theorem 7.1. Let $\kappa$ be infinite. There is a maximal antichain of cardinality $\kappa$ and a maximal antichain of cardinality $2^{\kappa}$ in $\Pi_{\kappa}$.

Proof. Atoms of $\Pi_{\kappa}$ are singular partitions with the non-singleton block containing only two elements and are thus in bijection with the set of two-element subsets of $\alpha$, hence there are $\kappa$ atoms. By atomicity of $\Pi_{\kappa}$, atoms are mutually incomparable (they form an antichain) and every other non- $\perp$ element lies over an atom (the antichain is maximal). 
Similarly, co-atoms are two blocks partitions of the form $\{A, \kappa \backslash A\}$. By picking the subset not containing 0 , they are in bijection with non-empty subsets of $\kappa \backslash\{0\}$, hence there are $2^{\kappa}$ co-atoms. By co-atomicity of $\Pi_{\kappa}$, co-atoms form a maximal antichain.

Since $\Pi_{\kappa}$ itself has cardinality $2^{\kappa}$, there is no antichain of a greater size. The proof that there is no maximal antichain shorter than $\kappa$ is more invoved.

Theorem 7.2. For infinite $\kappa$, no non-trivial maximal antichain in $\Pi_{\kappa}$ has cardinality less than $\kappa$.

Proof. Let $\lambda<\kappa$ and $\mathbf{A}=\left\{\mathcal{A}_{\delta} \mid \delta<\omega_{\lambda}\right\}$ be a non-trivial antichain of cardinality $\lambda$ in $\Pi_{\kappa}$. We will show that $\mathbf{A}$ is not maximal by building a partition $\mathcal{P} \notin \mathbf{A}$ that is not comparable to any of its elements.

We first consider the case where $\lambda$ is infinite.

Small partitions. For each $\mathcal{A}_{\delta}$, we build the set $S_{\delta}$ of all the elements that are not in a singleton block in $\mathcal{A}_{\delta}$ :

$$
S_{\delta}=\left\{x \in \kappa \mid \text { There exists } y \neq x \text { with } x \equiv_{\mathcal{A}_{\delta}} y\right\}
$$

Now, we call a partition $\mathcal{A}_{\delta}$ small if $\left|S_{\delta}\right| \leq \lambda$, i.e. there are less than $\lambda$ elements in all the non-singleton blocks of $\mathcal{A}_{\delta}$. Given an antichain $\mathbf{A}$, either it contains some small partitions or not.

No small partitions. Suppose that A contains no small partitions. Since it is not trivial, we know that $\top \notin \mathbf{A}$. Hence, for each $\mathcal{A}_{\delta} \in \mathbf{A}$ we can pick an element $x_{\delta}$ which is not in the same block as 0 , i.e. $0 \not \mathcal{A}_{\delta} x_{\delta}$. Note that the $x_{\delta}$ are not necessarily distinct. Now, build the singular partition $\mathcal{P}$ whose non-singleton block is $P_{0}=\{0\} \cup\left\{x_{\delta} \mid \delta<\omega_{\lambda}\right\}$. We claim that $\mathcal{P} \notin \mathbf{A}$ but $\mathbf{A} \cup\{\mathcal{P}\}$ is still an antichain, hence $\mathbf{A}$ is not maximal.

Because $P_{0}$ has cardinality at most $\lambda$ and all the remaining blocks are singletons, $\mathcal{P}$ is small and hence not in $\mathbf{A}$ by assumption. Moreover, it is not possible that $\mathcal{A}_{\delta} \leq$ $\mathcal{P}$. Indeed, because $\mathcal{P}$ contains only one non-singleton block, $P_{0}$, the comparison can hold only if $P_{0}$ contains $S_{\delta}$, the union of all the non-singleton blocks of $\mathcal{A}_{\delta}$. But $S_{\delta}$ has cardinality larger than $\lambda$ (because $\mathcal{A}_{\delta}$ is not small) while $P_{0}$ has cardinality at most $\lambda$. Hence, $S_{\delta} \not \subset P_{0}$ and $\mathcal{A}_{\delta} \not \leq \mathcal{P}$.

Next, for all $\delta<\omega_{\lambda}$ we have $0 \equiv_{\mathcal{P}} x_{\delta}$ by construction, but $0 \not \equiv \mathcal{A}_{\delta} x_{\delta}$, so $\mathcal{P} \not \subset \mathcal{A}_{\delta}$. Thus, $\mathbf{A} \cup\{\mathcal{P}\}$ is an antichain, and hence $\mathbf{A}$ was not maximal.

With small partitions. We now look at the case where $\mathbf{A}$ does contain small partitions. Let $\Delta$ be the set of indices of small partitions: $\Delta=\left\{\delta|| S_{\delta} \mid \leq \lambda\right\}$. Note that by construction, since $|\mathbf{A}|=\lambda$, we have $|\Delta| \leq \lambda$. Finally, let $S$ be the union of all non-singleton blocks among all the small partitions in A: $S=\bigcup_{\delta \in \Delta} S_{\delta}$.

It follows from cardinal arithmetic that $|S| \leq \lambda$ :

$$
|S|=\left|\bigcup_{\delta \in \Delta} S_{\delta}\right| \leq \sum_{\delta \in \Delta}\left|S_{\delta}\right| \leq \sum_{\delta \in \Delta} \lambda=|\Delta| \times \lambda \leq \lambda \times \lambda=\lambda
$$

The construction proceeds as follows: Since $|S| \leq \lambda<\kappa$, we have $|\kappa \backslash S|=\kappa$. Thus, in $\kappa \backslash S$, we can pick $\lambda$ different elements $y_{\delta}$, one for each $\delta<\omega_{\lambda}$. We claim that for each $\delta<\omega_{\lambda}$ we can find a $x_{\delta} \in S$ with $x_{\delta} \not \equiv \mathcal{A}_{\delta} y_{\delta}$. Indeed, if that were not the case then the block of $\mathcal{A}_{\delta}$ containing $y_{\delta}$ would also contain the entirety of $S$ and thus any of the small partitions in $\mathbf{A}$ would be smaller than $\mathcal{A}_{\delta}$, contradicting the fact that $\mathbf{A}$ is an antichain.

Note that the $y_{\delta}$ are distinct by hypothesis and that $x_{\delta} \neq y_{\delta}$, because the former is in $S$ and the latter is not. However, it is possible that the $x_{\delta}$ are not distinct.

We now build the equivalence (and partition) $\mathcal{Q}$ generated by the relation $\left\{\left(x_{\delta}, y_{\delta}\right) \mid \delta<\omega_{\lambda}\right\}$. Because the $x_{\delta}$ are not necessarily distinct, $\mathcal{Q}$ may contain blocks with more than 
two elements; indeed transitivity may link some of the pairs together. However, because the $y_{\delta}$ are distinct, the separation of every $x_{\delta}$ and $y_{\delta}$ ensures that we know each non-singleton block of $\mathcal{Q}$ to contain exactly one $x_{\delta}$ and one or more $y_{\delta}$.

For every $\delta<\omega_{\lambda}$ we have by construction $x_{\delta} \equiv_{\mathcal{Q}} y_{\delta}$, but $x_{\delta} \not \equiv \mathcal{A}_{\delta} y_{\delta}$. It follows from this both that $\mathcal{Q} \notin \mathbf{A}$, and that $\mathcal{Q} \not \leq \mathcal{A}_{\delta}$ for every $\mathcal{A}_{\delta} \in \mathbf{A}$.

We now prove non-maximality of $\mathbf{A}$ by showing that the extension $\mathbf{A} \cup\{\mathcal{Q}\}$ is an antichain. Consider first the case when $\mathcal{A}_{\delta}$ is small. By construction of $S$, there is at least one non-singleton block of $\mathcal{A}_{\delta}$ that is contained in $S$ (as $S$ is precisely the union of such blocks for all the small partitions). On the other hand, each non-singleton block of $\mathcal{Q}$ contains exactly one $x_{\delta}$ and all its other elements are $y_{\delta}$, chosen from $\kappa \backslash S$. Hence, each block of $\mathcal{Q}$ contains at most one element of $S$ and thus contains no non-singleton block of $\mathcal{A}_{\delta}$, whereby $\mathcal{A}_{\delta} \not \leq \mathcal{Q}$.

Second, consider the case when $\mathcal{A}_{\delta}$ is not small. By construction, the union of all the non-singleton blocks of $\mathcal{Q}$ is the set $\left\{x_{\delta} \mid \delta<\omega_{\lambda}\right\} \cup\left\{y_{\delta} \mid \delta<\omega_{\lambda}\right\}$, of cardinality $\leq \lambda$, while the union of all non-singleton blocks of $\mathcal{A}_{\delta}$ has cardinality $>\lambda$ (as it is not a small partition). Thus, it is not possible for the non-singleton blocks of $\mathcal{A}_{\delta}$ to be contained in the non-singleton blocks of $\mathcal{Q}$, and we conclude $\mathcal{A}_{\delta} \not \mathbf{L}$. Thus, $\mathcal{Q}$ is not in the antichain and is not comparable with its elements, hence $\mathbf{A}$ is not maximal.

Finite antichains. We finally turn to the case where $\lambda=n$ is finite. The proof is nearly the same as in the infinite case. We now call a partition $\mathcal{A}_{\delta}$ small if the union of its non-singleton blocks is finite. The proof in the case of no small $\mathcal{A}_{\delta}$ is the same with $\mathcal{P}_{0}=\left\{0, x_{1}, \ldots, x_{n}\right\}$ finite, hence $\mathcal{P}$ is small. For the case where $\mathbf{A}$ contains small partitions, since there are only finitely many of them, $\Delta$ is finite, hence $S$ is also finite. Therefore, the union of all non-singleton blocks of $\mathcal{Q}$ is finite, and the rest of the proof holds.

In conclusion, any antichain of cardinality strictly less than $\kappa$ is not maximal, so any maximal antichain in $\Pi_{\kappa}$ has cardinality at least $\kappa$.

Note that, while we here restrict our attention to infinite partition lattices, for finite $n$, maximal non-trivial antichains in $\Pi_{n}$ can be proved to have cardinality at least $n$ by induction on $n$.

Theorems 7.1 and 7.2 tell us that any maximal antichain in $\Pi_{\kappa}$ has cardinality between $\kappa$ and $2^{\kappa}$ and since both of the bounds occur as cardinality of a maximal antichain, they are as tight as possible. However, the following construction shows that, in some models where $\mathrm{GCH}$ is violated, there are maximal antichains of cardinalities between these bounds. Specifically, it is true whenever there exists $\lambda<\kappa<2^{\lambda}<2^{\kappa}$.

Remark 7.3. Let $\lambda<\kappa$ be two infinite cardinals. Given a maximal antichain $\mathbf{A}$ in $\Pi_{\lambda}$, we can construct a maximal antichain $\mathbf{B}$ of cardinality $\max \left(\kappa, 2^{\lambda}\right)$ in $\Pi_{\kappa}$ as follows:

For each partition $\mathcal{A} \in \mathbf{A}$, let $\mathcal{A}^{\prime}$ be a partition of $\kappa$ constructed from $\mathcal{A}$ by adding all the singleton blocks $\{x\}, x \in \kappa \backslash \lambda$ and let $\mathbf{A}^{\prime}$ be the collection of all these. Next, let $\mathcal{P}_{\alpha, \beta}$ be the singular partition of $\Pi_{\kappa}$ with non-singleton block $\{\alpha, \beta\}$ and define

$$
\mathbf{B}=\mathbf{A}^{\prime} \cup\left\{\mathcal{P}_{\alpha, \beta} \mid \alpha<\beta<\omega_{\kappa} \text { and } \lambda \leq|\beta|\right\}
$$

$\mathbf{B}$ is an antichain. Indeed, the $\mathcal{A}^{\prime}$ are mutually incomparable because $\mathbf{A}$ is an antichain; the $\mathcal{P}_{\alpha, \beta}$ are mutually incomparable by construction; and a $\mathcal{A}^{\prime}$ is not comparable with a $\mathcal{P}_{\alpha, \beta}$ because each of the non-singleton blocks of the former is included in $\lambda$ while the non-singleton block of the latter contains an element of $\kappa \backslash \lambda$ (as we have only taken the $\beta$ with $|\beta| \geq \lambda$ ). 
B is maximal. Indeed, consider a partition $\mathcal{Q}^{\prime}$ of $\Pi_{\kappa}$ which is not in B. If the elements of $\kappa \backslash \lambda$ are all in singleton blocks in $\mathcal{Q}^{\prime}$ then, by maximality of $\mathbf{A}$, its restriction $\mathcal{Q}=\mathcal{Q}^{\prime} \sqcap \lambda$ is comparable with some $\mathcal{A}$, and so $\mathcal{Q}^{\prime}$ is comparable with $\mathcal{A}^{\prime}$. On the other hand, if there is a non singleton block containing $\alpha, \beta$ with $\beta \in \kappa \backslash \lambda$ we immediately have $\mathcal{P}_{\alpha, \beta} \leq \mathcal{Q}$.

Because $\lambda<\kappa$, there are $|\kappa \backslash \lambda|=\kappa$ different $\beta$ with $\lambda \leq|\beta|$, thus there are $\kappa$ different $\mathcal{P}_{\alpha, \beta}$ in $\mathbf{B}$. Because the $\mathcal{A}^{\prime}$ and $\mathcal{P}_{\alpha, \beta}$ are distinct, $\mathbf{B}$ has cardinality $\left|\mathbf{A}^{\prime}\right|+\kappa=\max (\kappa,|\mathbf{A}|)$. Since we know, by Theorem 7.1. that we can build a maximal antichain of cardinality $2^{\lambda}$ in $\Pi_{\lambda}$, we can use this construction to build maximal antichains of cardinality $\max \left(\kappa, 2^{\lambda}\right)$ in $\Pi_{\kappa}$, for any value of $\lambda<\kappa$.

Thus, for every cardinality $\lambda$ such that $\lambda<\kappa<2^{\lambda}$, we can build an antichain with cardinality $2^{\lambda}$ with $\kappa<2^{\lambda}<2^{\kappa}$.

We do not yet know whether all cardinalities between the bounds always occur as antichains. Certainly, we can construct non-GCH models where there exist cardinals between $\kappa$ and $2^{\kappa}$ that cannot be written as $2^{\lambda}$. It is not yet known whether they can be realized as the cardinality of a maximal antichain through another construction.

\section{Complements}

Recall that in a bounded lattice $L$, elements $a, b \in L$ are complements if and only if $a \vee b=\top$ and $a \wedge b=\perp$. We denote by $\operatorname{compl}(\mathcal{P})$ the set of all complements to $\mathcal{P}$ in $\Pi_{\kappa}$. For finite $\kappa=n$, counting the number of elements in $\operatorname{compl}(\mathcal{P})$ is a difficult combinatorial problem. The best known estimate, due to Grieser Gri91, is that if $\mathcal{P}=\left\{B_{1}, \ldots, B_{m}\right\}$ is a partition in $\Pi_{n}$, then the number of complements $\mathcal{Q}$ of $\mathcal{P}$ satisfying $|\mathcal{Q}|=n-m+1$ is $\prod_{i=1}^{m}\left|B_{i}\right| \cdot(n-m+1)^{m-2}$.

In the following, we prove a succession of lemmas leading up to the main result of the section, Theorem 8.7, which gives a complete characterization of the counts of complements to partitions of infinite cardinals.

For later use, we first recall some fundamental results on cardinal arithmetic:

Lemma 8.1. Let $\left(\kappa_{i}\right)_{i \in I}$ be a family of cardinals. The following hold:

(1) ([HSW99], Lemma 1.6.3(b.i)) If $\kappa_{i}>0$ for all $i \in I, I \neq \emptyset$ and at least one of the cardinals $|I|$ and $\kappa_{i}$ (for some $i \in I$ ) is infinite, then

$$
\sum_{i \in I} \kappa_{i}=\max \left\{|I|, \sup \left\{\kappa_{i} \mid i \in I\right\}\right\}=|I| \cdot \sup \left\{\kappa_{i} \mid i \in I\right\}
$$

(2) ([HSW99], Lemma 1.6.15(a), Tarski), If $\lambda \geq \aleph_{0}$ is a cardinal and $\left(\kappa_{\alpha}\right)_{\alpha<\lambda}$ is an increasing sequence of infinite cardinals, then

$$
\prod_{\alpha<\lambda} \kappa_{\alpha}=\left(\sup \left\{\kappa_{\alpha} \mid \alpha<\lambda\right\}\right)^{\lambda}
$$

(3) ([HSW99], Lemma 1.6.15(d), Tarski) If $\kappa$ is an infinite cardinal, then

$$
2^{\kappa}=\left(\sup _{\lambda<\kappa} 2^{\lambda}\right)^{\operatorname{cf}(\kappa)}
$$

Lemma 8.2. Let $\kappa$ be an infinite cardinal, and $\mathcal{P} \notin\{\perp, \top\}$ be a partition of $\kappa$. Then, $|\operatorname{compl}(\mathcal{P})| \geq 2^{|\mathcal{P}|}$.

Proof. If $|\mathcal{P}|$ is finite, then there must exist at least one block $B$ of cardinality $\kappa$ in $\mathcal{P}$ (otherwise, since $\cup \mathcal{P}=\kappa$, we would have $\operatorname{cf}(\kappa)$ finite). Any singular partition whose non-singleton block contains exactly one element from each block of $\mathcal{P}$ is a complement to $\mathcal{P}$. Since there are $\kappa$ choices for the element in $B$, there are at least $\kappa$ complements to $\mathcal{P}$. Thus $|\operatorname{compl}(\mathcal{P})| \geq \kappa>2^{|\mathcal{P}|}$. 
Assume now that $|\mathcal{P}|$ is infinite. Since $\mathcal{P} \neq \perp$, we can choose a block $B_{0}$ from $\mathcal{P}$ containing distinct elements $\iota \neq v$. Write $\mathcal{P}^{\prime}=\mathcal{P} \backslash\left\{B_{0}\right\}$, and note that $\left|\mathcal{P}^{\prime}\right|=|\mathcal{P}|$. By the Axiom of Choice, we may select from each block $B \in \mathcal{P}^{\prime}$ an element $\gamma(B)$. Given any subset $\mathcal{P}_{1} \subseteq \mathcal{P}^{\prime}$, let $\mathcal{P}_{2}=\mathcal{P}^{\prime} \backslash \mathcal{P}_{1}$. If we now define

$$
\begin{aligned}
& Q_{1}=\{\iota\} \cup\left\{\gamma(B) \mid B \in \mathcal{P}_{1}\right\} \\
& Q_{2}=\{v\} \cup\left\{\gamma(B) \mid B \in \mathcal{P}_{2}\right\} \\
& \mathcal{Q}_{s}=\left\{\{\gamma\} \mid \gamma \in \kappa \backslash\left(Q_{1} \cup Q_{2}\right)\right\}
\end{aligned}
$$

then $\mathcal{Q}=\left\{Q_{1}, Q_{2}\right\} \cup \mathcal{Q}_{s}$ is a complement to $\mathcal{P}$, as can be verified as follows: (i) $\mathcal{Q}$ is a partition, since each element is included in exactly one block. (ii) $\mathcal{P} \wedge \mathcal{Q}=\perp$, since each block $Q \in \mathcal{Q}$ contains at most one element from each block in $\mathcal{P}$. (iii) Finally, $\mathcal{P} \vee \mathcal{Q}=\top$, i.e. $x \equiv \mathcal{P} \vee \mathcal{Q} y$ for all $x, y \in \kappa$ : Consider $x, y \in \kappa$, and write $B_{x}, B_{y}$ for the blocks in $\mathcal{P}$ containing $x$ and $y$, respectively. If $B_{x}, B_{y} \in \mathcal{P}_{1} \cup\left\{B_{0}\right\}$, then there exists ${ }^{3}$ a $\gamma_{x} \in Q_{1} \cap B_{x}$ and a $\gamma_{y} \in Q_{1} \cap B_{y}$. This yields

$$
x \underset{\overline{B_{x}}}{\equiv} \gamma_{x} \overline{\overline{\overline{Q_{1}}}} \gamma_{y} \overline{\overline{\overline{B_{y}}}} y
$$

If instead $B_{x} \in \mathcal{P}_{1} \cup\left\{B_{0}\right\}$ and $B_{y} \in \mathcal{P}_{2}$, there is a $\gamma_{x} \in Q_{1} \cap B_{x}$, whereby

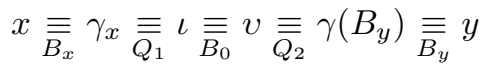

The remaining two cases, $B_{x}, B_{y} \in \mathcal{P}_{2} \cup\left\{B_{0}\right\}$ and $B_{x} \in \mathcal{P}_{2} \cup\left\{B_{0}\right\}, B_{y} \in \mathcal{P}_{1}$, are symmetrical. Thus $\mathcal{Q} \in \operatorname{compl}(\mathcal{P})$. Clearly, two different choices of the subset $\mathcal{P}_{1} \subseteq \mathcal{P}^{\prime}$ yields different complements $\mathcal{Q}$, whereby $|\operatorname{compl}(\mathcal{P})| \geq 2^{|\mathcal{P}|}$.

Lemma 8.3. Let $\kappa$ be an infinite cardinal, and $\mathcal{P} \notin\{\perp, \top\}$ be a partition of $\kappa$. If $\mathcal{P}$ contains no block of cardinality $\kappa$, then $|\operatorname{compl}(\mathcal{P})|=2^{\kappa}$

Proof. If $\mathcal{P}$ contains no block of cardinality $\kappa$, then $|\mathcal{P}| \geq \operatorname{cf}(\kappa)$ (because $\kappa=$ $\left.\bigcup_{B \in \mathcal{P}} B\right)$. Then Lemma 8.2 implies $|\operatorname{compl}(\mathcal{P})| \geq 2^{|\mathcal{P}|} \geq 2^{\operatorname{cf}(\kappa)}$. Thus, if $\kappa$ is a regular cardinal, or if $|\mathcal{P}|=\kappa$, we immediately obtain $|\operatorname{compl}(\mathcal{P})|=2^{\kappa}$.

Assume now that $\kappa$ is singular and $\operatorname{cf}(\kappa) \leq|\mathcal{P}|<\kappa$. We can construct a complement $\mathcal{Q}$ to $\mathcal{P}$ as follows:

(1) Let $A_{0}$ be a set containing exactly one element $\gamma(B)$ from each block $B$ of $\mathcal{P}$. Let $\mathcal{P}^{\prime}=\left\{B \backslash A_{0} \mid B \in \mathcal{P}\right\} \backslash\{\emptyset\}$, whereby $\cup \mathcal{P}^{\prime}=\kappa \backslash A_{0}$.

(2) Any partition $\mathcal{Q}$ of $\kappa$ that contains $A_{0}$ as a block will have $\mathcal{P} \vee \mathcal{Q}=\top$ : If $\beta \in B_{1}$ and $\delta \in B_{2}$ for $B_{1}, B_{2} \in \mathcal{P}$, then $\beta \equiv_{B_{1}} \gamma\left(B_{1}\right) \equiv_{A_{0}} \gamma\left(B_{2}\right) \equiv_{B_{2}} \delta$. Hence, if a partition $\mathcal{Q}^{\prime}$ of $\kappa \backslash A_{0}$ satisfies $\left|A^{\prime} \cap B^{\prime}\right| \leq 1$ for all $A^{\prime} \in \mathcal{Q}^{\prime}, B^{\prime} \in$ $\mathcal{P}^{\prime}$, then $\mathcal{Q}=\left\{A_{0}\right\} \cup \mathcal{Q}^{\prime}$ is a complement to $\mathcal{P}$.

(3) Because $\left|A_{0}\right|=|\mathcal{P}|<\kappa$, we have $\left|\kappa \backslash A_{0}\right|=\kappa$, and hence $\sum_{B \in \mathcal{P}^{\prime}}|B|=$ $\left|\cup \mathcal{P}^{\prime}\right|=\kappa$. By Lemma 8.1]1, and using $\left|\mathcal{P}^{\prime}\right|<\kappa$, we obtain

$$
\kappa=\sum_{B \in \mathcal{P}^{\prime}}|B|=\max \left\{\left|\mathcal{P}^{\prime}\right|, \sup _{B \in \mathcal{P}^{\prime}}|B|\right\}=\sup _{B \in \mathcal{P}^{\prime}}|B|
$$

(4) By definition of cofinality, there exists an increasing sequence $\left(\mu_{\alpha}\right)_{\alpha<\operatorname{cf}(\kappa)}$ of infinite cardinals strictly less than $\kappa$ that sums to $\kappa$. Because $\kappa$ is singular, Lemma 8.111 implies $\kappa=\sup _{\alpha<\operatorname{cf}(\kappa)} \mu_{\alpha}$.

Since also $\kappa=\sup \left\{|B| \mid B \in \mathcal{P}^{\prime}\right\}$, we can choose by AC for every $\alpha<\operatorname{cf}(\kappa)$ some $B_{\alpha} \in \mathcal{P}^{\prime}$ such that $\left|B_{\alpha}\right| \geq \mu_{\alpha}$. The sequence $\left(\left|B_{\alpha}\right|\right)_{\alpha<\operatorname{cf}(\kappa)}$ clearly has supremum $\kappa$.

\footnotetext{
${ }^{3}$ Namely, $\gamma_{x}=\gamma\left(B_{x}\right)$ if $B_{x} \in \mathcal{P}_{1}$ and $\gamma_{x}=\iota$ if $B_{x}=B_{0}$.
} 
(5) For every successor ordinal $\alpha+1<\mathrm{cf}(\kappa)$, split the block $B_{\alpha+1}$ into a small subset $B_{\alpha+1}^{-}$of cardinality $\left|B_{\alpha}\right|$ and a large subset $B_{\alpha+1}^{+}$of cardinality $\left|B_{\alpha+1}\right|$. Then for every limit ordinal $\alpha$, define $B_{\alpha}^{+}=B_{\alpha}$. Now choose for every ordinal $\alpha<\operatorname{cf}(\kappa)$ a bijection $\sigma_{\alpha}: B_{\alpha}^{+} \rightarrow B_{\alpha+1}^{-}$; let $A_{\alpha}^{\beta}=\left\{\beta, \sigma_{\alpha}(\beta)\right\}$ and let $A_{\alpha}=\left\{A_{\alpha}^{\beta} \mid \beta \in B_{\alpha}^{+}\right\}$, it is a partition of $B_{\alpha}^{+} \cup B_{\alpha+1}^{-}$, consisting of two-element blocks.

(6) Finally, let

$$
S=\kappa \backslash\left(A_{0} \cup \bigcup_{\alpha<\operatorname{cf}(\kappa)} B_{\alpha}\right) .
$$

Then it is easy to verify that

$$
\mathcal{Q}=\{\{\beta\} \mid \beta \in S\} \cup\left\{A_{0}\right\} \cup \bigcup_{\alpha<\operatorname{cf}(\kappa)} A_{\alpha}
$$

is a complement to $\mathcal{P}$. Indeed, each element of $A_{\alpha}$ contains exactly two elements from two different blocks $B_{\alpha}$ and $B_{\alpha+1}$ of $\mathcal{P}^{\prime}$, and the other blocks are either $A_{0}$ or singletons. Thus, for any $A^{\prime} \in \mathcal{Q}$ other than $A_{0}$ and any $B^{\prime} \in \mathcal{P}^{\prime}$, we have $\left|A^{\prime} \cap B^{\prime}\right| \leq 1$, and by Point (2) above, $\mathcal{Q}$ is a complement to $\mathcal{P}$.

For $\sigma_{\alpha} \neq \sigma_{\alpha}^{\prime}$ there exists $\beta \in B_{\alpha}^{+}$with $\sigma_{\alpha}(\beta) \neq \sigma_{\alpha}^{\prime}(\beta)$ and hence $\left\{\beta, \sigma_{\alpha}(\beta)\right\} \neq$ $\left\{\beta, \sigma_{\alpha}^{\prime}(\beta)\right\}$, whence each choice of $\left(\sigma_{\alpha}\right)_{\alpha<\operatorname{cf}(\kappa)}$ yields a distinct complement. There are $\left.\left|B_{\alpha+1}^{-}\right|\right|^{+B_{\alpha} \mid}=\left|B_{\alpha}\right|^{\left|B_{\alpha}\right|}=2^{\left|B_{\alpha}\right|}$ ways to choose each bijection $\sigma_{\alpha}$. Since $\kappa=$ $\sup _{\alpha<\operatorname{cf}(\kappa)}\left|B_{\alpha}\right|$, for each $\lambda<\kappa$, there exists $\alpha$ with $\left|B_{\alpha}\right|>\lambda$ hence $2^{\left|B_{\alpha}\right|} \geq 2^{\lambda}$. Consequently, $\sup _{\alpha<\operatorname{cf}(\kappa)} 2^{\left|B_{\alpha}\right|} \geq \sup _{\lambda<\kappa} 2^{\lambda}$. Then, Lemma 8.12 and 3 yields

$$
|\operatorname{compl}(\mathcal{P})| \geq \prod_{\alpha<\operatorname{cf}(\kappa)} 2^{\left|B_{\alpha}\right|}=\left(\sup _{\alpha<\operatorname{cf}(\kappa)} 2^{\left|B_{\alpha}\right|}\right)^{\operatorname{cf}(\kappa)} \geq\left(\sup _{\lambda<\kappa} 2^{\lambda}\right)^{\operatorname{cf}(\kappa)}=2^{\kappa}
$$

As $2^{\kappa}=\left|\Pi_{\kappa}\right|$ is an upper bound to the number of complements to $\mathcal{P}$, we obtain $|\operatorname{compl}(\mathcal{P})|=2^{\kappa}$.

Lemma 8.4. Let $\kappa$ be an infinite cardinal, and $\mathcal{P} \in \Pi_{\kappa}$ be any partition of $\kappa$. If $\mathcal{P}$ contains a block $B$ of cardinality $\kappa$, then $|\operatorname{compl}(\mathcal{P})|=\kappa^{\lambda}$, where $\lambda=|\kappa \backslash B|$.

Proof. Assume that $\mathcal{P}$ has a block $B$ of cardinality $\kappa$, and write $\mathcal{P}$ as the disjoint union $\mathcal{P}=\{B\} \cup \mathcal{P}^{\prime}$. Let $\bar{B}=\kappa \backslash B$, and observe that $\bar{B}=\cup \mathcal{P}^{\prime}$. Denote $\lambda=\left|\cup \mathcal{P}^{\prime}\right|=$ $|\bar{B}|=|\kappa \backslash B| \leq \kappa$. If $|\bar{B}|=0$, then $\mathcal{P}=\top$, which has exactly one complement, namely $\perp$, whereby $|\operatorname{compl}(\mathcal{P})|=1=\kappa^{0}$ as desired. Hence, in the following we can assume that $\mathcal{P} \neq \top$, such that $1 \leq \lambda \leq \kappa$.

Lower bound. To show $|\operatorname{compl}(\mathcal{P})| \geq \kappa^{\lambda}$, choose any injection $\sigma: \bar{B} \rightarrow B$, and write $B_{\lambda}=\sigma(\bar{B}), B_{s}=B \backslash B_{\lambda}$. If we now define $A_{\alpha}=\{\alpha, \sigma(\alpha)\}$, then the partition $\mathcal{Q}=\left\{A_{\alpha} \mid \alpha \in \bar{B}\right\} \cup\left\{\{\beta\} \mid \beta \in B_{s}\right\}$ is a complement to $\mathcal{P}$, as is easily verified by checking each of the properties: (i) Every element of $\kappa$ is either in $\bar{B}$, $B_{\lambda}$, or $B_{s}$, hence $\mathcal{Q}$ is a partition; (ii) each block of $\mathcal{Q}$ is either a singleton (from $B_{s}$ ) or a doubleton (one of the $A_{\alpha}$ ) with one element in $B$ and one out, hence it intersects each block of $\mathcal{P}$ in at most one point; (iii) any $\alpha$ and $\alpha^{\prime}$ are linked through their images $\sigma(\alpha)$ and $\sigma\left(\alpha^{\prime}\right)$ in $B$.

There are $\kappa^{\lambda}$ ways of choosing $\sigma$, and each way leads to a distinct complement. Hence, $|\operatorname{compl}(\mathcal{P})| \geq \kappa^{\lambda}$.

Upper bound. Observe that we have $|\bar{B}| \geq\left|\mathcal{P}^{\prime}\right|$. Let now $\mathcal{Q}$ be any complement to $\mathcal{P}$. Then 
(1) $\mathcal{P} \wedge \mathcal{Q}=\perp$ implies that for every $A \in \mathcal{Q}$ and $B \in \mathcal{P},|A \cap B| \leq 1$, and hence $|A| \leq|\mathcal{P}| \leq \lambda$.

(2) Let $\mathcal{Q}^{\prime}=\left\{A^{\prime} \in \mathcal{Q}|| A^{\prime} \mid \geq 2\right\}$ be the set of non-singleton blocks of $\mathcal{Q}$. Since $\left|A^{\prime} \cap B\right| \leq 1$, each $A^{\prime}$ must intersect $\bar{B}$ in at least one point. As blocks of a partition, the $A^{\prime}$ are pairwise disjoint, as are consequently these intersections. Hence choosing (by $\mathrm{AC}$ ) an element in each $A^{\prime} \cap \bar{B}$ defines an injection from $\mathcal{Q}^{\prime}$ to $\bar{B}$, whereby $\left|\mathcal{Q}^{\prime}\right| \leq \bar{B} \leq \lambda$.

(3) An upper bound for the number of complements to $\mathcal{P}$ can then be found in the following way: Specifying $\mathcal{Q}^{\prime}$ uniquely determines the complement $\mathcal{Q}$. $\mathcal{Q}$ has $\left|\mathcal{Q}^{\prime}\right| \leq \lambda$ non-singleton blocks, each of size at most $|\mathcal{P}| \leq \lambda$, yielding $\left|\bigcup \mathcal{Q}^{\prime}\right| \leq|\lambda \times \lambda|=\lambda$. A complement $\mathcal{Q}$ is fully specified by (i) the union $\bigcup \mathcal{Q}^{\prime}$ of the non-singleton blocks; and (ii) the partition of these unions into blocks of $\mathcal{Q}^{\prime}$. Write $\epsilon=\left|\bigcup \mathcal{Q}^{\prime}\right|$. For each cardinality $\epsilon$ that $\bigcup \mathcal{Q}^{\prime}$ can attain, there are at most $\lambda^{\epsilon} \leq \kappa^{\epsilon}$ ways to select the elements $\bigcup \mathcal{Q}^{\prime}$ from $\bar{B}$. Since $\mathcal{Q}^{\prime}$ is a partition of $\epsilon$, there are at most $\left|\Pi_{\epsilon}\right|=2^{\epsilon}$ distinct ways to partition these elements into blocks of $\mathcal{Q}^{\prime}$. Letting now $\epsilon$ range over all potentially allowed cardinalities, i.e. all less than or equal to $\lambda$, we find

$$
|\operatorname{compl}(\mathcal{P})| \leq \sum_{\epsilon \leq \lambda} 2^{\epsilon} \cdot \lambda^{\epsilon} \leq \sum_{\epsilon \leq \lambda} 2^{\epsilon} \cdot \kappa^{\epsilon}=\sum_{\epsilon \leq \lambda} \kappa^{\epsilon}=\kappa^{\lambda}
$$

The above applies both to finite and infinite $\lambda=|\kappa \backslash B|$.

Corollary 8.5. Let $\kappa$ be infinite and $\mathcal{P} \notin\{\perp, \top\}$ be a partition of $\kappa$. If $\mathcal{P}$ contains two or more blocks of cardinality $\kappa$, then $|\operatorname{compl}(\mathcal{P})|=2^{\kappa}$.

Proof. If two blocks, $B_{1}$ and $B_{2}$, have cardinality $\kappa$, then $\left|\kappa \backslash B_{1}\right|=\kappa$, and Lemma 8.4 yields $|\operatorname{compl}(\mathcal{P})| \geq \kappa^{\kappa}=2^{\kappa}$.

Lemma 8.6. Let $\kappa$ be infinite and $\mathcal{P} \notin\{\perp, \top\}$ be a partition of $\kappa$. Then, $\kappa \leq$ $|\operatorname{compl}(\mathcal{P})| \leq 2^{\kappa}$.

Proof. As $\left|\Pi_{\kappa}\right|=2^{\kappa}$, the upper bound is immediate, and it suffices to prove the lower bound. There are two cases to consider: First, if $\mathcal{P}$ contains no block of cardinality $\kappa$, Lemma 8.3 yields $|\operatorname{compl}(\mathcal{P})|=2^{\kappa}$. Otherwise, if $\mathcal{P}$ does contain a block $B$ of cardinality $\kappa$, Lemma 8.4 implies $|\operatorname{compl}(\mathcal{P})|=\kappa^{|\kappa \backslash B|}$. Because $\mathcal{P} \neq \top$, we have $|\kappa \backslash B| \geq 1$, whereby $|\operatorname{compl}(\mathcal{P})| \geq \kappa$. In both cases, $|\operatorname{compl}(\mathcal{P})| \geq \kappa$.

We can now state our main theorem:

Theorem 8.7. Let $\kappa$ be infinite and $\mathcal{P} \notin\{\perp, \top\}$ be a partition in $\Pi_{\kappa}$. Then

(1) $\kappa \leq|\operatorname{compl}(\mathcal{P})| \leq 2^{\kappa}$.

(2) $|\operatorname{compl}(\mathcal{P})| \geq 2^{|\mathcal{P}|}$.

(3) If $\mathcal{P}$ contains no block of cardinality $\kappa$, then $|\operatorname{compl}(\mathcal{P})|=2^{\kappa}$.

(4) If $\mathcal{P}$ contains a block $B$ of cardinality $\kappa$, then $|\operatorname{compl}(\mathcal{P})|=\kappa^{|\kappa \backslash B|}$.

(5) If $\mathcal{P}$ contains two or more blocks of cardinality $\kappa$, then $|\operatorname{compl}(\mathcal{P})|=2^{\kappa}$.

Proof. (1)-(4) are Lemmas 8.6, 8.2, 8.3 and 8.4, respectively. (5) is Corollary 8.5 .

A consequence of Theorem 8.7 is that partitions with fewer complements than $2^{\kappa}$ must always have exactly one large block, and a sufficiently small number of elements remaining after removing it:

Corollary 8.8. If $|\operatorname{compl}(\mathcal{P})|<2^{\kappa}$, then $\mathcal{P}$ contains exactly one block $B$ of size $\kappa$, and $|\kappa \backslash B|<\kappa$. 
For any infinite cardinal $\kappa$, both $\kappa$ and $2^{\kappa}$ can be realized as $|\operatorname{compl}(\mathcal{P})|$ for some partition $\mathcal{P}$. In fact, Theorem 8.7 provides a complete characterization of the cardinals that can be realized as complement counts: it is precisely those cardinals of the form $\kappa^{\lambda}$, with $0 \leq \lambda \leq \kappa$ (and $1 \leq \lambda \leq \kappa$ when considering only non-trivial partitions), as the following corollary shows:

Corollary 8.9. For any infinite cardinality $\kappa$, and every cardinal $0 \leq \lambda \leq \kappa$, there is a partition $\mathcal{P}_{\lambda}$ of $\kappa$ for which $\left|\operatorname{compl}\left(\mathcal{P}_{\lambda}\right)\right|=\kappa^{\lambda}$.

In particular, there exist partitions $\mathcal{P}$ and $\mathcal{R}$ in $\Pi_{\kappa}$ with $|\operatorname{compl}(\mathcal{P})|=\kappa$, respectively $|\operatorname{compl}(\mathcal{R})|=2^{\kappa}$.

No cardinal that is not of the form $\kappa^{\lambda}$ can be realised as $|\operatorname{compl}(\mathcal{P})|$.

Proof. Theorem 8.7 fully describes the possible number of complements of nontrivial partition. Remembering that $\kappa^{\kappa}=2^{\kappa}$ and that, for $\top$ and $\perp$, the number of complements is $1=\kappa^{0}$, it is apparent that for any partition, the number of complements has the form $\kappa^{\lambda}$. Conversely, the cardinal $\kappa^{\lambda}, 1 \leq \lambda \leq \kappa$, can be realised as the number of complements to the two-block partition $\{B, \bar{B}\}$ with $|B|=\lambda$ and $|\bar{B}|=\kappa$, using Theorem 8.7(4).

8.1. Orthocomplements. It is well-known that $\Pi_{\kappa}$ is relatively complemented. To our knowledge, it has so far been unknown whether there exists an $n>2$ such that $\Pi_{n}$ is orthocomplemented. We prove that for any cardinality $\kappa>2$ (finite or transfinite), $\Pi_{\kappa}$ is not orthocomplemented.

Proposition 8.10 (Orthocomplements). If $\kappa>2$, then $\Pi_{\kappa}$ is not orthocomplemented.

Proof. Orthocomplementation yields a bijection between atoms and co-atoms. Atoms are singular partitions whose non-singleton block is a pair, hence there are as many atoms as there are pairs, namely $\frac{\kappa \times(\kappa-1)}{2}$. Co-atoms are partitions with two blocks, a non-trivial subset of $\kappa$ and its complement, hence there are as many co-atoms as half the number of non-trivial subsets, namely $\frac{2^{\kappa}-2}{2}$. For $\kappa \geq 3$, these numbers are different hence $\Pi_{\kappa}$ cannot be orthocomplemented. For $\kappa=3$, one can easily check that $\Pi_{3}$ is not orthocomplemented either (because it contains an odd number of elements).

\section{Results Under GCH}

Under the Generalized Continuum Hypothesis, the results from the previous sections all simplify greatly, and it is possible to obtain much stronger results.

9.1. Maximal chains under GCH. Under GCH, we can fully determine the possible cardinals for maximal chains in $\Pi_{\kappa}$, as we will see in Theorem 9.4 below.

Lemma 9.1. Let $\kappa$ be an infinite cardinal, let $\mathbf{C}$ be a maximal chain in $\Pi_{\kappa}$. Given a partition $\mathcal{P} \in \mathbf{C}$, write $\mathcal{P}^{+}=\wedge \mathbf{C}_{\mathcal{P}}^{+}$(cf. Definition 3.1), and let

$$
\mathbf{C}^{*}=\left\{\mathcal{P} \in \mathbf{C} \mid \mathcal{P} \prec \mathcal{P}^{+}\right\}
$$

Then $\kappa \leq 2^{\left|\mathbf{C}^{*}\right|}$.

Proof. First note that, being maximal, $\mathbf{C}$ is end-point including, closed and covering by Lemma 3.3. Let $\mathcal{P}_{\alpha, \beta}^{-}=\vee \mathbf{C}_{\alpha, \beta}^{-}$(cf. Definition 3.4. By Lemma 3.5. we have $\mathcal{P}_{\alpha, \beta}^{-} \prec \wedge \mathbf{C}_{\alpha, \beta}^{+}$and by construction, $\mathbf{C}_{\alpha, \beta}^{+}=\mathbf{C}_{\mathcal{P}_{\alpha, \beta}^{-}}^{+}$. Hence, $\mathcal{P}_{\alpha, \beta}^{-} \in \mathbf{C}^{*}$.

We next construct a family of maps $\varphi_{\alpha}: \mathbf{C}^{*} \rightarrow\{0,1\}$ in the following way: Let $\mathcal{P} \in \mathbf{C}^{*}$. By definition, we have $\mathcal{P} \prec \mathcal{P}^{+}$, thus there exist an unique block $B_{\mathcal{P}}$ of $\mathcal{P}^{+}$which is the union of two blocks of $\mathcal{P}$ (and all others are also blocks of $\mathcal{P}$ ). Choose, by axiom of choice, one of these two blocks as $B_{\mathcal{P}, 0}$. 
Now, for each $\alpha \in \kappa$, we define $\varphi_{\alpha}: \mathbf{C}^{*} \rightarrow\{0,1\}$ by

$$
\varphi_{\alpha}(\mathcal{P})= \begin{cases}0 & \text { if } \alpha \in B_{\mathcal{P}, 0} \\ 1 & \text { otherwise }\end{cases}
$$

The previous construction shows that for any $\alpha \neq \beta$, (i) $\mathcal{P}_{\alpha, \beta}^{-} \in \mathbf{C}^{*}$; (ii) $\alpha$ and $\beta$ are precisely in $B_{\mathcal{P}_{\alpha, \beta}^{-}}$; and (iii) they are in different blocks of $\mathcal{P}_{\alpha, \beta}^{-}$, that is one of them is in $B_{\mathcal{P}_{\alpha, \beta}^{-}, 0}$ and the other is not. Hence, $\varphi_{\alpha}\left(\mathcal{P}_{\alpha, \beta}^{-}\right) \neq \varphi_{\beta}\left(\mathcal{P}_{\alpha, \beta}^{-}\right)$and consequently, $\varphi_{\alpha} \neq \varphi_{\beta}$. Thus, the map $\alpha \mapsto \varphi_{\alpha}$ is injective from $\kappa$ to $\{0,1\}^{\mathbf{C}^{*}}$, whereby $\kappa \leq 2^{\left|\mathbf{C}^{*}\right|}$.

Proposition 9.2. Let $\kappa$ be any infinite cardinal. Under GCH, any maximal chain C in $\Pi_{\kappa}$ has cardinality

$$
|\mathbf{C}| \geq \begin{cases}\kappa^{-} & \text {if } \kappa \text { is a successor cardinal, } \\ \kappa & \text { if } \kappa \text { is a limit cardinal. }\end{cases}
$$

Proof. By Lemma 9.1, $\kappa \leq 2^{\left|\mathbf{C}^{*}\right|} \leq 2^{|\mathbf{C}|}$ for any maximal chain C. Under GCH, $|\mathbf{C}|^{+}=2^{|\mathbf{C}|}$, whereby $\kappa \leq|\mathbf{C}|^{+}$. Assume now that $|\mathbf{C}|<\kappa$, i.e. $|\mathbf{C}|<\kappa \leq|\mathbf{C}|^{+}$. By definition of the successor relation, this implies $\kappa=|\mathbf{C}|^{+}$.

If $\kappa$ is a limit cardinal, this is a contradiction, and so we must have $|\mathbf{C}| \geq \kappa$. If $\kappa$ is a successor cardinal, it follows that $|\mathbf{C}| \geq \kappa^{-}$.

By combining the previous results, we can tightly bound the cardinal of any maximal chain in $\Pi_{\kappa}$ under GCH. In addition, the following simple restriction of Theorems 5.2 and 6.14 to the case when $\mathrm{GCH}$ is assumed, provides instances of long and short maximal chains that realize the bounds established above.

Corollary 9.3. Let $\kappa$ be a infinite cardinal. Under $G C H$, there exists a maximal chain of length $\kappa^{+}$in $\Pi_{\kappa}$; and there exists a chain of length $\kappa$ in $\Pi_{\kappa^{+}}$.

Proof. If GCH is assumed, the first statement is an immediate consequence of Theorem 5.2 and the second statement follows, because the precondition in Theorem 6.14, $\lambda<\kappa$ only if $2^{\lambda}<2^{\kappa}$, is always satisfied under GCH.

Theorem 9.4. Let $\kappa$ be an infinite cardinal. Under $G C H$, the cardinality of any maximal chain in $\Pi_{\kappa}$ is:

- $\kappa^{-}, \kappa$, or $\kappa^{+}$(and all three are always achieved) if $\kappa$ is a successor; and

- either $\kappa$ or $\kappa^{+}$(and both are achieved) if $\kappa$ is a limit cardinal.

Proof. The lower bounds are given by Proposition 9.2, and the upper bound is the cardinality of $\Pi_{\kappa}$ itself.

Furthermore, each possible value is always realized: For any infinite cardinal $\kappa$, there always exists a well-founded maximal chain of cardinality $\kappa$. By Corollary 9.3 , there also exists a chain of length $\kappa^{+}$, and for successor cardinals $\kappa$, a chain of length $\kappa^{-}$.

The bounds can also be stated in a symmetrical fashion:

Corollary 9.5. For any infinite cardinal $\kappa$ (successor or limit), the cardinality of any maximal chain lies between $\sup \{\lambda<\kappa\}$ and $\inf \{\lambda>\kappa\}$.

\subsection{Maximal antichains under GCH.}

Corollary 9.6. Under $G C H$, when $\kappa$ is an infinite cardinal, the length of any maximal antichain in $\Pi_{\kappa}$ is either $\kappa$ or $\kappa^{+}$, and both are realized.

Proof. This is the content of Theorems $7.1,7.2$ together with the fact that $\left|\Pi_{\kappa}\right|=$ $2^{\kappa}$, when the assumption $2^{\kappa}=\kappa^{+}$is made. 
9.3. Complements under GCH. Under $\mathrm{GCH}$, the number of complements $|\operatorname{compl}(\mathcal{P})|$ for partitions $\mathcal{P} \notin\{\top, \perp\}$ of an infinite cardinal $\kappa$ is either $\kappa$ or $\kappa^{+}=2^{\kappa}$. In addition, the simplified rules for arithmetic under GCH strengthen Theorem 8.7

Theorem 9.7. Let $\kappa$ be an infinite cardinal, and $\mathcal{P} \notin\{\top, \perp\}$ be a partition of $\kappa$. Assuming GCH, then

$$
|\operatorname{compl}(\mathcal{P})|= \begin{cases}\kappa & \text { if and only if exactly one block } B \in \mathcal{P} \text { has }|B|=\kappa, \\ & \text { and }|\kappa \backslash B|<\operatorname{cf}(\kappa) \\ 2^{\kappa} & \text { otherwise }\end{cases}
$$

Proof. Consider the three cases:

(1) First, if $\mathcal{P}$ contains either zero or at least two blocks of size $\kappa$, then Theorem 8.7 (2) or 8.7 (5) yields $|\operatorname{compl}(\mathcal{P})|=2^{\kappa}$.

(2) Next, assume $\mathcal{P}$ contains exactly one block $B$ of size $\kappa$, and $|\kappa \backslash B| \geq \operatorname{cf}(\kappa)$. By Theorem 8.7 $(4),|\operatorname{compl}(\mathcal{P})|=\kappa^{|\kappa \backslash B|} \geq \kappa^{\operatorname{cf}(\kappa)}>\kappa$. GCH, together with $|\operatorname{compl}(\mathcal{P})| \leq 2^{\kappa}$, then yields $|\operatorname{compl}(\mathcal{P})|=2^{\kappa}$.

(3) Finally, if $\mathcal{P}$ contains exactly one block $B$ of size $\kappa$ in $\mathcal{P}$, and $|\kappa \backslash B|<\operatorname{cf}(\kappa)$. Under GCH, $\kappa^{\lambda}=\kappa$ if and only if $1 \leq \lambda<\operatorname{cf}(\kappa)$. Together with Theorem 8.7 (4) and $\mathcal{P} \neq \top$, this yields $|\operatorname{compl}(\mathcal{P})|=\kappa^{|\kappa \backslash B|}=\kappa$.

In Theorem 9.7, the assumption of GCH is necessary in the sense that it is easy to construct non-GCH examples that violate the result. For example, consider a model where $2^{\aleph_{1}}=2^{\aleph_{2}}=\aleph_{3}$, which is consistent with ZFC by Easton's Theorem, and let $\kappa=\aleph_{2}$. Consider a partition $\mathcal{P}=\left\{B, B^{\prime}\right\}$, where $|B|=\aleph_{2}$, and $\left|B^{\prime}\right|=\aleph_{1}<\operatorname{cf}\left(\aleph_{2}\right)$. Then $|\kappa \backslash B|=\left|B^{\prime}\right|=\aleph_{1}$, so $8.7(4)$ yields $|\operatorname{compl}(\mathcal{P})|=\aleph_{2}^{\aleph_{1}} \geq 2^{\aleph_{1}}$. But in this model, $2^{\aleph_{1}}=2^{\aleph_{2}}=2^{\kappa}>\kappa$, despite $|\kappa \backslash B|<\operatorname{cf}(\kappa)$.

However, the result does hold for some classes of cardinals, regardless of whether GCH is assumed. In particular, whenever $\mu^{\lambda}<\kappa$ for all $\mu<\kappa$ and all $\lambda<\operatorname{cf}(\kappa)$. This is for example the case when $\kappa$ is a strong limit cardinal.

9.4. Gathering all results under GCH. Collecting the results of this section gives us the following concise characterization of chains, antichains, and complements in infinite partition lattices under $\mathrm{GCH}$ :

Theorem 9.8. Under GCH, when $\kappa$ is an infinite cardinal:

(1) Any maximal well-founded chain in $\Pi_{\kappa}$ always has cardinality $\kappa$.

(2) Any general maximal chain in $\Pi_{\kappa}$ has cardinality

(a) $\kappa^{-}, \kappa$, or $\kappa^{+}$(and all three are always achieved) if $\kappa$ is a successor cardinal; and

(b) either $\kappa$ or $\kappa^{+}$(and both are achieved) if $\kappa$ is a limit cardinal.

(3) Any non-trivial maximal antichain in $\Pi_{\kappa}$ has cardinality either $\kappa$ or $\kappa^{+}$, and both are achieved.

(4) Any non-trivial partition has either $\kappa$ or $\kappa^{+}$complements. $\mathcal{P} \notin\{\perp, \top\}$ has $\kappa$ complements if and only if $(i) \mathcal{P}$ contains exactly one block, $B$, of cardinality $\kappa$, and (ii) $|\kappa \backslash B|<\mathrm{cf}(\kappa)$; otherwise, $\mathcal{P}$ has $\kappa^{+}$complements.

\section{ACKnowledgments}

The authors thank Benoît Kloeckner for his many comments on a preliminary version of this article, and thank the anonymous referees for valuable suggestions. James Avery was supported by VILLUM FONDEN through the network for Experimental Mathematics in Number Theory, Operator Algebras, and Topology. 
Jean-Yves Moyen was partially supported by the ANR project "Elica" ANR-14CE25-0005 and by the Marie Skłodowska-Curie action "Walgo", program H2020MSCA-IF-2014, number 655222. Pavel Ruzicka was partially supported by the Grant Agency of the Czech Republic under the grant no. GACR 14-15479S. Jakob Grue Simonsen was partially supported by the Danish Council for Independent Research Sapere Aude grant "Complexity through Logic and Algebra" (COLA).

\section{REFERENCES}

[BH02] Vladimir Markovich Blinovsky and Lawrence Hueston Harper. Size of the largest antichain in a partition poset. Problems of Information Transmission, 38(4):347-353, 2002.

[Bir40] Garrett Birkhoff. Lattice Theory, volume 25 of Colloquium Publications. American Mathematical Society, 1940.

[Can98] E. Rodney Canfield. The size of the largest antichain in the partition lattice. Journal of Combinatorial Theory, Series A, 83(2):188 - 201, 1998.

[Czé96a] Gábor Czédli. Four-generated large equivalence lattices. Acta. Sci. Math. (Szeged.), 62:47-69, 1996

[Czé96b] Gábor Czédli. Lattice generation of small equivalences of a countable set. Order, 13(1):11-16, 1996

[Czé99] Gábor Czédli. (1+1+2)-generated equivalence lattices. Journal of Algebra, 221(2):439462, 1999.

[Eas70] William B. Easton. Powers of regular cardinals. Annals of Mathematical Logic, 1(2):139 $-178,1970$.

[Grä03] George Grätzer. General Lattice Theory. Birkhäuser, second edition, 2003.

[Gri91] Daniel Grieser. Counting complements in the partition lattice, and hypertrees. Journal of Combinatorial Theory, Series A, 57(1):144-150, 1991.

[Har05] Egbert Harzheim. Ordered Sets, volume 7 of Advances in Mathematics. Springer-Verlag, second edition, 2005.

[Hau14] Felix Hausdorff. Grundzüge der Mengenlehre. Leipzig, 1914.

[HSW99] M. Holz, K. Steffens, and E. Weitz. Introduction to Cardinal Arithmetic. Birkhäuser Verlag, 1999.

[Kön05] Julius König. Über die Grundlage der Mengenlehre und das Kontinuumproblem. Mathematische Annalen, 61:156-160, 1905.

[Ore42] Øystein Ore. Theory of equivalence relations. Duke Mathematical Journal, 9(3):573-627, 1942.

[RS92] Ivan Rival and Miriam Stanford. Algebraic aspects of partition lattices. In Neil White, editor, Matroid Applications, volume 40 of Encyclopedia of Mathematics and its Applications, pages 106-122. Cambridge University Press, 1992.

[Sie22] Wacław Franciszek Sierpiński. Sur un problème concernant les sous-ensembles croissants du continu. Fundamenta Mathematicae, 3:109-112, 1922. 